\title{
1,3-硒唑为模板多种杂环修饰的新型分子的设计、合成及活性研究
}

\author{
张成路* 孙晓娜蒲雨昕李传银 \\ 孙丽杰王 静李益政 \\ (辽宁师范大学化学化工学院 大连 116029)
}

\begin{abstract}
摘要 以取代 1,3 -硒唑为模板, 分别利用 $1,2,4$-三唑、四唑、噁二唑、吡唑、1,2,4-三嗪和丁二酰亚胺等修饰, 首次设计 合成了 6 类共 22 个目标分子, 并利用 IR, NMR 和 HRMS 等波谱技术对目标分子进行了结构表征. 评价了目标分子对 $\mathrm{Cdc} 25 \mathrm{~B}$ 的抑制活性, 结果发现, 13 个目标分子具有较好的抑制活性, 其中有 5 种目标分子的抑制活性高于阳性参照物 $\mathrm{Na}_{3} \mathrm{VO}_{4}$ 有望成为抗肿瘤药物先导物. 构效分析结果发现, 在 1,3-硒唑骨架上引入多氮杂环三唑、四唑和三嗪, 引入含 有氨基和颈基的噻二唑和啞二唑等有望获得活性优良的新型含硒有机分子.
\end{abstract}

关键词 1,3-硒唑; 多杂环; Cdc25B 抑制剂

\section{Design, Synthesis and Activities of Multiheterocyclic Modified Novel Molecules Using 1,3-Selenazole as Template}

\author{
Zhang, Chenglu* Sun, Xiaona Pu, Yuxin Li, Chuanyin \\ Sun, Lijie Wang, Jing Li, Yizheng \\ (College of Chemistry and Chemical Engineering, Liaoning Normal University, Dalian 116029)
}

\begin{abstract}
Six kinds, twenty-two novel target molecules were first designed and synthesized by using substituted 1,3-selenazole as a template, which were modified by 1,2,4-triazole, tetrazole, oxadiazole, pyrazole, 1,2,4-triazine and succinic imide respectively. Their structures were confirmed by IR, NMR and HRMS. The inhibitory activities of the target molecules against cell division cycle 25B phosphatase (Cdc25B) were evaluated. As a result, thirteen compounds exhibited good inhibitory activities. The $\mathrm{IC}_{50}$ values of five compounds were lower than the positive reference $\mathrm{Na}_{3} \mathrm{VO}_{4}$ and were expected to be anticancer drugs leading compounds. The analysis of structure-activity relationship found that the introduction of multinitrgon-heterocyclic triazole, tetrazole and triazine, thiadiazole and oxadiazole containing amino or mercapto group onto 1,3-selenazole were expected to obtain novel excellent bioactivity organic selenium containing molecules.
\end{abstract}

Keywords 1,3-selenazole; multi-heterocycle; Cdc25B inhibitor

噁唑和噻唑是众多天然产物和临床药物中重要的 药效基团. 含噁唑和噻唑的分子具有广泛的生物活性, 如抗菌 ${ }^{[1 \sim 3]}$ 和抗肿瘤 ${ }^{[47]}$ 等. 该类分子在生化反应中易 代谢, 且本质是非致癌的 ${ }^{[8]}$, 因此具有噁唑和噻唑分子 的设计合成已成为药物篮选的重要途径. 硒是人体必需 微量元素, 是重要的食物源抗氧化剂, 缺硒会引起如癌 症、心脏病、关节炎和免疫系统紊乱等疾病, 流行病学 调查结果表明, 适量补硒有益于人体健康等 ${ }^{[9,10]}$. 无其 是近年来的研究结果表明, 有机硒化物具有优良的抗肿 瘤细胞增殖活性，是蛋白激酶活化剂、抗氧化剂，具有
抗炎和抗菌活性 ${ }^{[11 ~ 13]}$ 等, 因此新型有机硒化物的研究 引起了广泛的关注. 由于硒、硫和氧元素为同族元素, 理化性质相似, 用硒元素替代噻唑和噁唑结构中的硫元 素和氧元素, 一方面可为人体补充必须的微量元素硒, 另一方面根据生物电子等排原理, 可以保持分子的构型 和电子云分布基本不变, 同时硒元素的引入可以影响有 机分子的化学性质并提高其生物活性，如噻唑呋喃类似 物硒唑呋喃(图 1), 在体外及体内的抗肿瘤活性篮选中 均优于噻唑呋喃 ${ }^{[14]}$, 表明 1,3 -硒唑具有更为优异的化学 和生物学特性, 因此设计合成含有硒唑结构基元的新型

\footnotetext{
* Corresponding author. E-mail: zhangchenglu@lnnu.edu.cn

Received July 15, 2016; revised September 3, 2016; published online October 9, 2016.

Project supported by the Science and Technology Research Program of Liaoning Provincial Department of Education (No. 2009A426).

辽宁省教育厅科学技术研究(No. 2009A426)资助项目.
} 
分子，探究不同结构硒唑衍生物的合成策略，无疑具有 重要的理论创新意义, 并为篎选活性优良的硒唑衍生物 奠定基础.
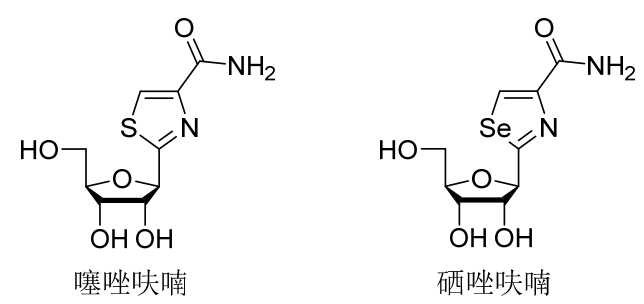

图 1 含有噻唑呋喃和硒唑呋喃的分子

Figure 1 Molecules containing thiazole furan and selenazole furan

在药用活性分子的研究中, 含不同杂环结构分子的 设计合成已成为药物创制的重要方法, 其中吡唑、三唑、 噁二唑、噻唑和四唑等活性结构的引入, 已成为药用分 子设计的重中之重. 吡唑衍生物因其优良的抗肿瘤活 性 ${ }^{[15 ~ 17]}$ 等, 显示了化学治疗的潜力; 三唑衍生物因具有 杀虫、除草、抗菌、促进和调节农作物生长功效, 以及 抗炎、抗病毒和抗肿瘤等生物活性, 已在农业和医药等 领域得到了广泛的应用 ${ }^{[18-23]} ; 1,3,4$-哑二唑衍生物也表

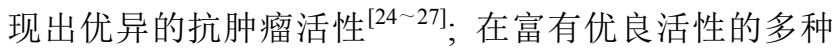
天然产物和大量与医学和生物学特性相关的有意义的 药物中, 噻唑是其核心结构, 该类分子能有效治疗高血 压、精神分裂症、炎症、过敏和 HIV 感染细菌等 症 ${ }^{[28-34]}$; 含四唑杂环的分子在抗癌、抗血压、抗过敏和 抗菌性等方面得到广泛的应用, 使四唑类药物合成篮选 具有很大的发展潜能 ${ }^{[35 ~ 37]}$; 近年来, 环状亚胺类化合物 因其广泛生物活性 ${ }^{[38 \sim 43]}$, 结构的多样性和广谱的生物 活性已被确定为最有前途的骨架, 成为学科领域的研究 热点 ${ }^{[44]}$.

为了构筑以硒唑为核心骨架的含不同杂环的新型 分子, 研究同一模板下不同杂环药效基团同存于一分子 中的合成策略, 本文以 2-对甲氧苯基-1,3-硒唑为模板, 将吡唑、三唑、噁二唑、噻唑和四唑等优良药效杂环结 构通过经典的方法共筑于不同分子中, 创建 22 个不同 结构的新型杂环分子, 并通过其对细胞分裂雕期蛋白 (cell division cycle 25, 缩写为 Cdc25B) 双位点磷酸酷酶 抑制作用，对不同结构的分子进行抗癌活性评价.

\section{1 结果与讨论}

\section{1 目标化合物的合成与表征}

通过 ${ }^{1} \mathrm{H} N M R 、{ }^{13} \mathrm{C} N M R$ 、IR 和 HRMS 等波谱技术 对 22 种目标分子进行结构表征: 在 IR 谱图中, 3100 和 $1600 \sim 1450 \mathrm{~cm}^{-1}$ 的吸收峰表明目标分子的结构中存在
芳香环. 在 ${ }^{1} \mathrm{H} N \mathrm{NMR}$ 测试中, $\delta 7.00 \sim 8.00$ 出现芳香环的 质子信号, 在 $\delta 3.8$ 左右处出现对甲氧基的特征峰, 在 $\delta$ 2.7 左右处出现与硒唑环相连接的甲基的特征峰. 在 ${ }^{13} \mathrm{C}$ NMR 谱图中, 在 $\delta 110 \sim 185$ 范围内出现的信号峰为芳 香环和硒唑环的碳信号, 在 $\delta 55$ 左右为对甲氧基碳信号 峰, 在 $\delta 18.2$ 左右为硒唑环上甲基的碳信号峰. 在 HRMS 谱图中, 目标分子均出现了 $[\mathrm{M}+1]^{+}$峰. 目标分 子的合成路线如 Schemes 1 7 所示.

\section{2 目标化合物的生物活性测试}

应用大肠杆菌表达系统表达 Cdc25B 催化结构域, 以 GST 融合蛋白形式存在于清液中, 细胞裂解后经 GSH-亲和柱纯化, 获得活性 GST 融合的 Cdc25B 蛋白. 采用荧光底物 OMFP 考察不同化合物对重组酶的活性 抑制情况. OMFP 水解产物 OMF 被 $485 \mathrm{~nm}$ 激发光激发 后可发射出波长为 $530 \mathrm{~nm}$ 的荧光检测信号, 从而考察 酶的活性变化及化合物对其抑制情况.

目标分子对 Cdc25B 的抑制活性测试由国家新药篮 选中心完成. 在底物浓度为 $5 \mu \mathrm{g} / \mathrm{mL}$ 时, 设置三个复孔, 对样品的活性进行初篎测试. 选择抑制率大于 $50 \%$ 的化 合物进行复篎, 测试样品浓度为 $5 \mu \mathrm{g} / \mathrm{mL}$ 时的抑制活性, 得出抑制活性剂量依赖关系, 即 $\mathrm{IC}_{50}$ 值. $\mathrm{IC}_{50}$ 值通过样 品活性对样品浓度进行非线性拟和得到, 笁选实验方法 按照参考文献[45 47]的方法进行. 测试结果如表 1 所 示.

对 22 种含硒坐的杂环化合物进行了抑制 Cdc25B 的活性的研究, 结果表明, 13 种化合物对 Cdc25B 具有 良好的抑制活性，其中 SAZ-14 的抑制率最高, 达到 $105.04 \%$. 在进一步测试 $\mathrm{IC}_{50}$ 值的结果中, SAZ-1、

SAZ-9、SAZ-13、SAZ-14 和 SAZ-18 共有 5 种目标分 子的抑制活性高于阳性参照物 $\mathrm{Na}_{3} \mathrm{VO}_{4}[(1.86 \pm 0.24)$ $\mu \mathrm{g} / \mathrm{mL}]$, 其 $\mathrm{IC}_{50}$ 值分别为 $1.82 \pm 0.65 、 1.46 \pm 0.45 、 1.75$ $\pm 0.39 、 1.25 \pm 0.26$ 和 $1.46 \pm 0.19 \mu \mathrm{g} / \mathrm{mL}$. 分析化合物结 构发现，以对甲氧苯基取代的 1,3-硒唑为模板, 当在结 构中分别引入吡唑、含氨基或颈基的 1,2,4-三㟇、含酰 亚氨基的 1,3,4-噻二唑、含亚氨基的 1,3,4-噁二唑、1,2,4三嗪和顺丁二烯酰亚胺等时, 目标分子均具有较高的抑 制活性.

\section{2 结论}

由于 $\operatorname{Cdc} 25 \mathrm{~B}$ 的活性中心具有亲核基团，易受到亲 电子化合物的攻击. 已报道的一类亲电子抑制剂 ${ }^{[48]}$, 其 抑制作用产生的原因是通过它们的亲电子基团进攻活 性中心的半胱氨酸残基. 目标分子 SAZ-19 和 SAZ-21 中附加的环状酰亚胺基元是具有较好的亲电性, 因此表 

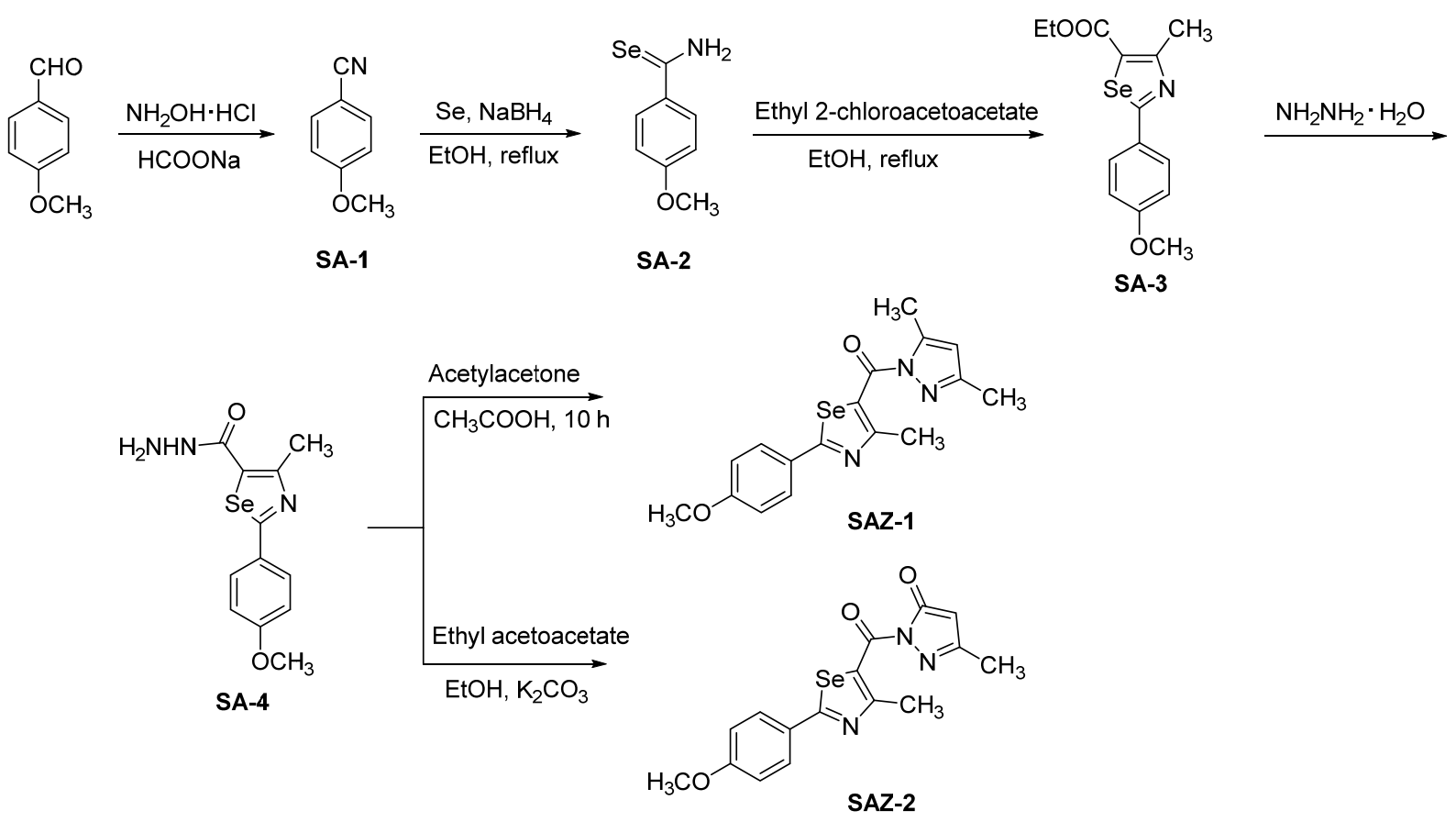

图式 1 合成含吡唑的化合物 SAZ-1 $\sim$ SAZ-2

Scheme 1 Synthesis of pyrazole containing compounds SAZ-1 $\sim$ SAZ-2

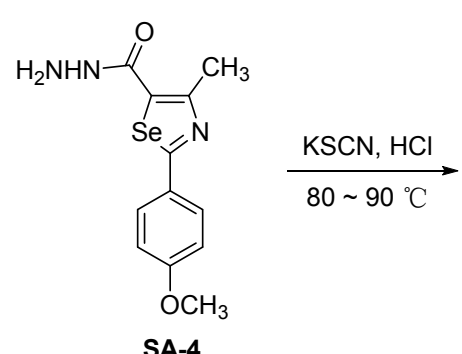

SA-4<smiles>COc1ccc(-c2nc(C)c(C(=O)NNC(N)=S)s2)cc1</smiles>

SA-7<smiles>COc1ccc(-c2nc(C)c(-c3n[nH]c(S)n3)[se]2)cc1</smiles>

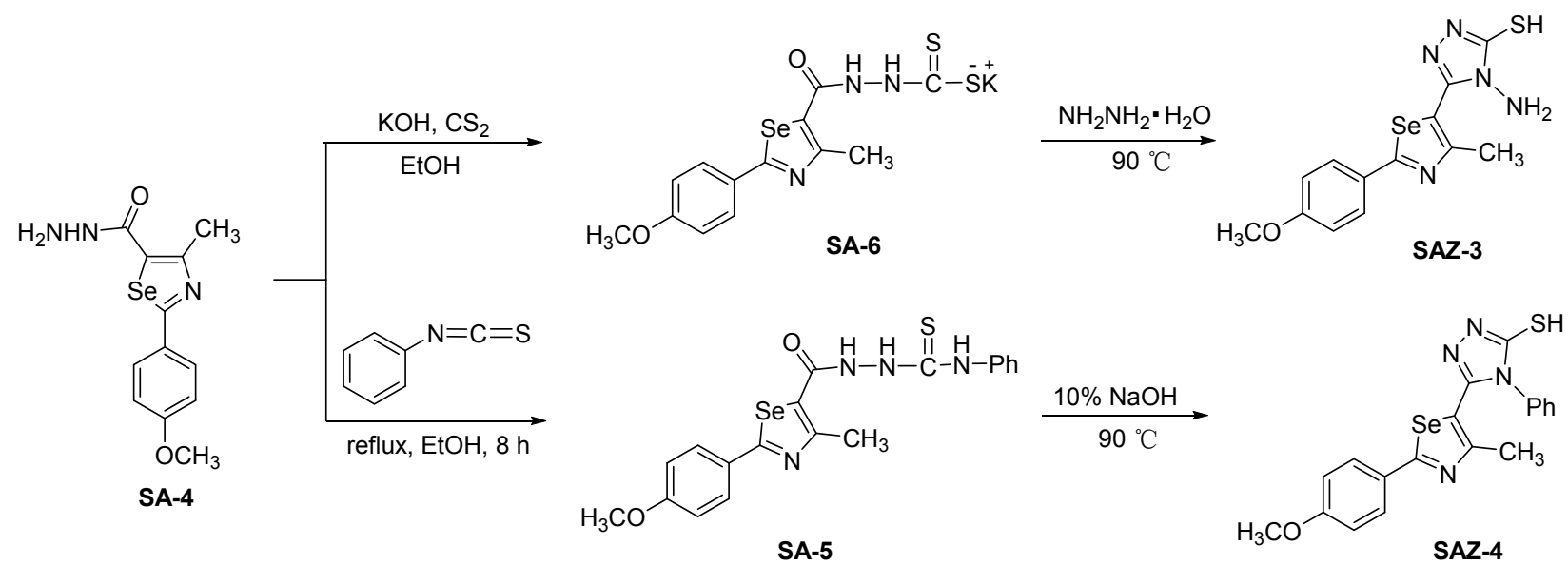

图式 2 合成含 1,2,4-三坐的化合物 SAZ-3 $\sim$ SAZ-5

Scheme 2 Synthesis of 1,2,4-triazole containing compounds SAZ-3 $\sim$ SAZ-5

达出优良的抑制活性. 在 SAZ-16、SAZ-17 和 SAZ-22 分子中, 虽然含有酰亚胺等亲电子基团, 但因其分子具 有较大的空间位阻, 加大了亲电基团与活性基团的靶点 的相互作用的难度, 所以表现出对 Cdc25B 的抑制活性
较低. 含多氮杂环 1,2,4-三唑 $(\mathbf{S A Z}-3 \sim \mathbf{S A Z}-5)$ 、四唑 (SAZ-18)和 1,2,4-三嗪(SAZ-20), 以及含吡唑(SAZ-18) 和噻二唑(SAZ-13 和 SAZ-14)药效基团的目标分子, 因 与生物大分子中杂原子作用位点增多, 可以引起细胞的 


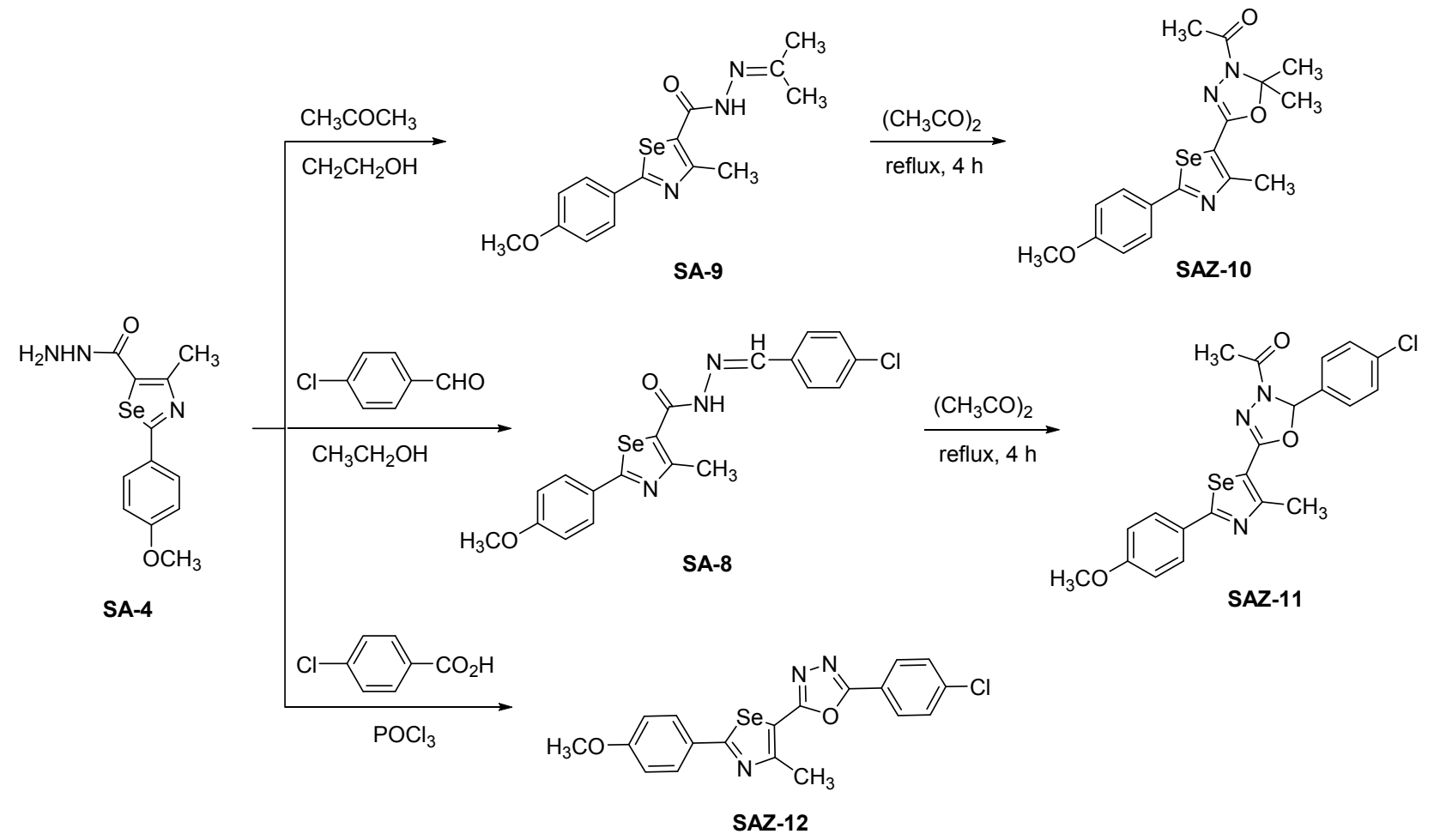<smiles>COc1ccc(-c2nc(C)c(C(=O)NN)s2)cc1</smiles>

图式 3 合成含噁二唑的化合物 SAZ 6 SAZ-12

Scheme 3 Synthesis of oxadiazole containing compounds SAZ 6 SAZ-12 
<smiles>COc1ccc(-c2nc(C)c(C(N)=O)s2)cc1</smiles>

SA-4<smiles>COc1ccc(-c2nc(C)c(-c3nnc(Nc4ccccc4)s3)[se]2)cc1</smiles><smiles>COc1ccc(-c2nc(C)c(C(=O)NN)s2)cc1</smiles>

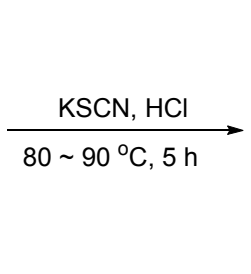<smiles>COc1ccc(-c2nc(C)c(C(=O)NNC(N)=S)s2)cc1</smiles><smiles>[Mg][Os]</smiles><smiles>COc1ccc(-c2nc(C)c(-c3nnc(N)s3)[se]2)cc1</smiles>

图式 4 合成含噻二唑的化合物 SAZ-13 SAZ-14

Scheme 4 Synthesis of thiadiazole containing compounds SAZ-13 $\sim$ SAZ-14<smiles>COc1ccc(C2NC(C)C(C(=O)NNC(=S)Nc3ccccc3)S2)cc1</smiles><smiles>COc1ccc(-c2nc(C)c(C(=O)N3C(=O)CS/C3=N\c3ccccc3)[se]2)cc1</smiles>

SA-5<smiles>COc1ccc(C2NC(C)C(C(=O)NNC(=S)Nc3ccccc3)[Se]2)cc1</smiles>

SA-5

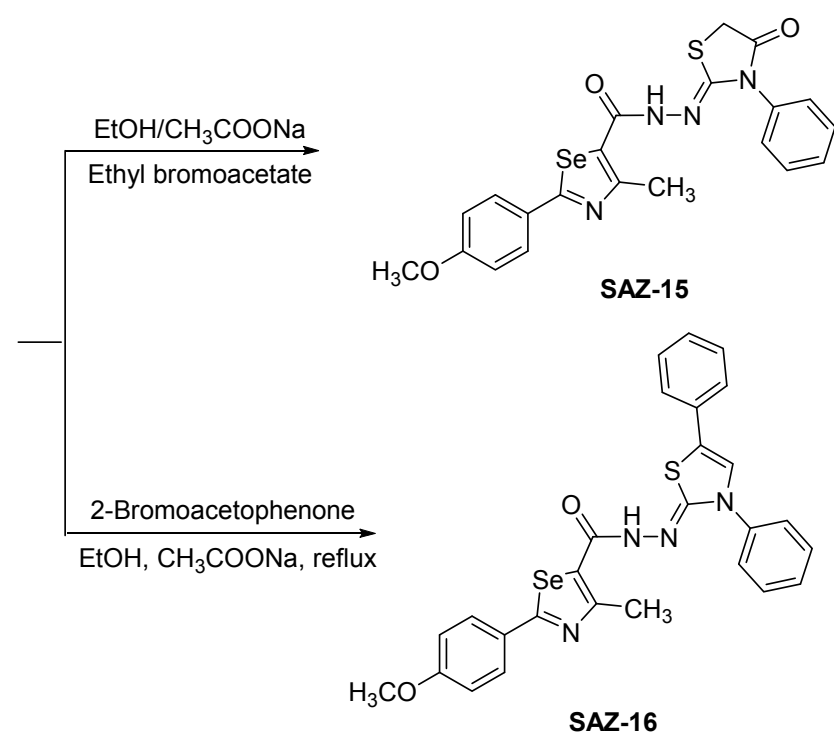

图式 5 合成含噻唑环的化合物 SAZ-15 SAZ-17

Scheme 5 Synthesis of the thiazole ring-containing compounds SAZ-15 $\sim$ SAZ-17 
<smiles>CCOC(=O)c1sc(-c2ccc(OC)cc2)nc1COc1ccc(-c2nc(C)c(C(N)=O)s2)cc1</smiles>

图式 6 合成含四唑环的化合物 SAZ-18

Scheme 6 Synthesis of the tetrazole ring-containing compounds SAZ-18

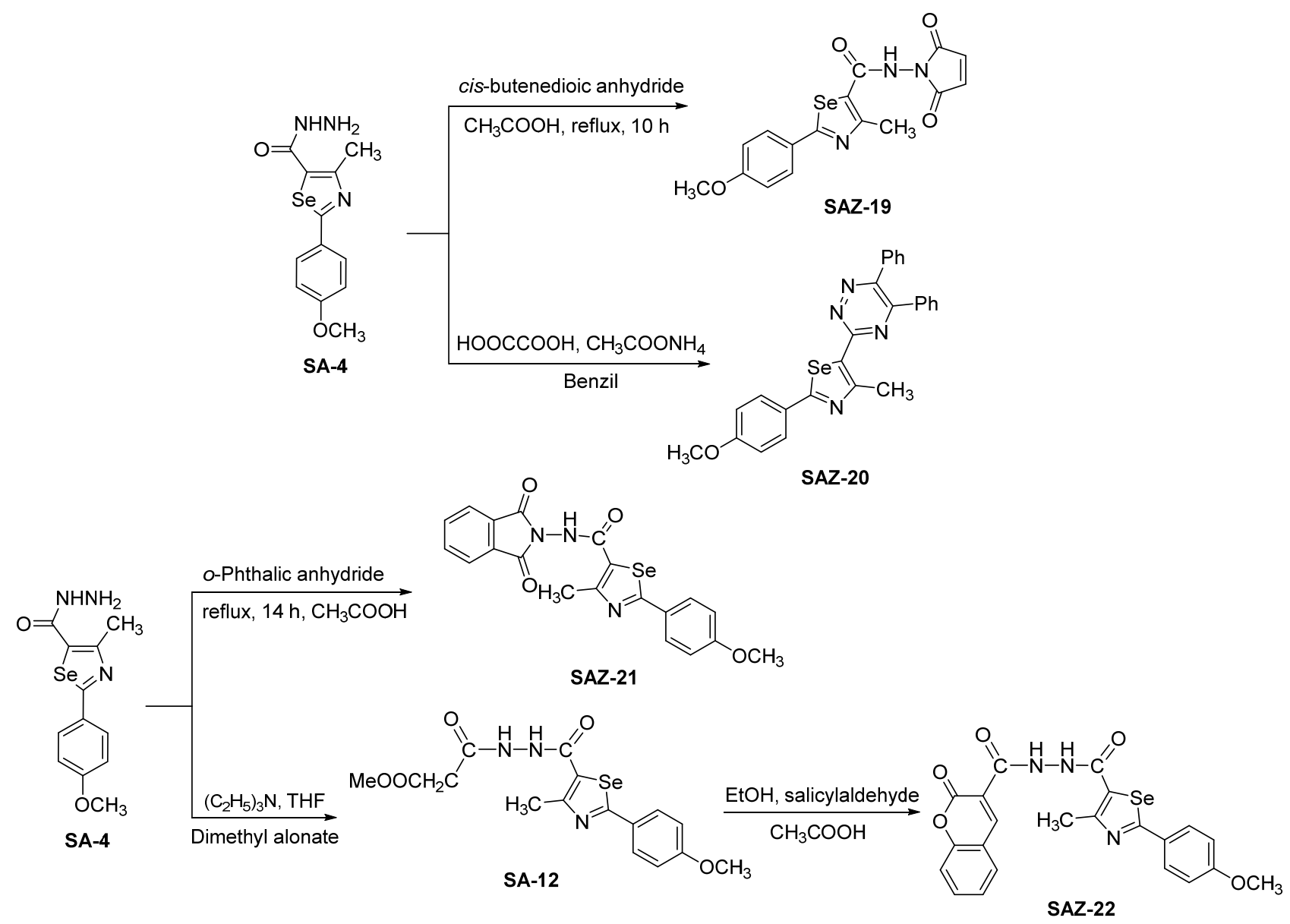

图式 7 合成其他杂环化合物 SAZ-19 SAZ-22

Scheme 7 Synthesis other heterocyclic compounds SAZ-19 $\sim$ SAZ-22

G2/M 期阻滞, 从而抑制细胞增殖, 达到较好的抗癌活 性效果. 值得关注的是含有氨基、颈基和苯亚氨基的目 标分子 SAZ-3 $\sim$ SAZ-5、SAZ-9、SAZ-13 和 SAZ-5 均 表现出优良的活性, 其可能原因是目标分子与生物大分 子之间产生了氢键的作用，使活性得到了提高.

综上所述, 芳基取代的 1,3-硒唑分别与吡唑、含氨 基或颈基的 12,4-三唑、含酰亚氨基的 1,3,4-噻二唑、含 苯亚氨基的 1,3,4-噁二唑、1,2,4-三嗪、四唑和酰亚胺等 拼合, 表现出较优良的抑制 Cdc25B 活性, 有望成为抗 肿瘤药物的先导物, 研究结果为创建和篮选新型硒唑药 物分子提供了重要的参考.

\section{3 实验部分}

\section{1 仪器与试剂}

对甲氧基苯甲醛、对氯苯甲醛、水杨醛、对氯苯甲 酸、硒粉、硼氢化钠、2-氯乙酰乙酸乙酯、乙酰乙酸乙 酯、原甲酸三乙酯、异硫氰酸苯酯、乙酰丙酮、氯乙酰 氯、2-溴苯乙酮、三氯氧磷、邻苯二甲酸酐、顺丁烯二 酸䣶、水合肼、二硫化碳、 $N, N$-羰基二咪唑(CDI)和叠氮 化钠(国药集团化学试剂有限公司), 所用试剂均为分析 纯.

AVANCE $500 \mathrm{MHz}$ NMR 核磁共振波谱仪; Accu- 
表 1 目标化合物对 Cdc25B 的抑制活性

Table 1 Inhibition of target compounds against Cdc25B

\begin{tabular}{|c|c|c|c|c|c|}
\hline \multirow{2}{*}{ Compd. } & \multicolumn{2}{|c|}{$\mathrm{Cdc} 25 \mathrm{~B}$} & \multirow{2}{*}{ Compd. } & \multicolumn{2}{|c|}{$\mathrm{Cdc} 25 \mathrm{~B}$} \\
\hline & Inhibition rate ${ }^{a} / \%$ & $\mathrm{IC}_{50}^{b} /\left(\mu \mathrm{g} \cdot \mathrm{mL}^{-1}\right)$ & & Inhibition rate ${ }^{a} / \%$ & $\mathrm{IC}_{50}^{b} /\left(\mu \mathrm{g} \cdot \mathrm{mL}^{-1}\right)$ \\
\hline SAZ-1 & $84.30 \pm 4.52$ & $1.82 \pm 0.65$ & SAZ-13 & $75.15 \pm 4.16$ & $1.75 \pm 0.39$ \\
\hline SAZ-2 & $78.06 \pm 7.06$ & $1.89 \pm 0.14$ & SAZ-14 & $105.04 \pm 2.10$ & $1.25 \pm 0.26$ \\
\hline SAZ-3 & $96.07 \pm 6.88$ & $1.95 \pm 0.34$ & SAZ-15 & $77.62 \pm 7.62$ & $3.25 \pm 0.45$ \\
\hline SAZ-4 & $87.07 \pm 0.46$ & $2.04 \pm 0.22$ & SAZ-16 & $9.48 \pm 3.41$ & NA \\
\hline SAZ-5 & $73.30 \pm 5.05$ & $4.85 \pm 0.29$ & SAZ-17 & $15.86 \pm 3.89$ & NA \\
\hline SAZ-6 & $18.8 \pm 3.32$ & NA & SAZ-18 & $90.57 \pm 6.85$ & $1.46 \pm 0.19$ \\
\hline SAZ-7 & $25.6 \pm 3.08$ & NA & SAZ-19 & $83.23 \pm 1.03$ & $3.93 \pm 0.16$ \\
\hline SAZ-8 & $31.99 \pm 3.13$ & NA & SAZ-20 & $74.21 \pm 2.45$ & $4.34 \pm 0.36$ \\
\hline SAZ-9 & $87.23 \pm 7.64$ & $1.46 \pm 0.45$ & SAZ-21 & $81.27 \pm 3.94$ & $2.66 \pm 0.14$ \\
\hline SAZ-10 & $28.77 \pm 4.56$ & NA & SAZ-22 & $51.53 \pm 6.14$ & NA \\
\hline SAZ-11 & $20.76 \pm 1.12$ & NA & $\mathrm{Na}_{3} \mathrm{VO}_{4}$ & - & $1.86 \pm 0.24$ \\
\hline SAZ-12 & $16.18 \pm 9.22$ & NA & & & \\
\hline
\end{tabular}

待测样品浓度为 $20 \mu \mathrm{g} / \mathrm{mL}$ 时的抑制率; ${ }^{b} \mathrm{IC}_{50}$ 值通过待测样品在浓度为 $5 \mu \mathrm{g} / \mathrm{mL}$ 时的抑制率非线性拟和计算得到

rate-Mass TOF LC/MS 型精确质量液相色谱飞行时间质 谱联用仪; TENSOR 27 傅立叶变换红外光谱仪; X-5 型 显微熔点测定仪, 温度未校正; WFH-203B 型三用紫外 分析仪.

\section{2 化合物 SA-1 SA-12 的合成}

中间体 SA-1 根据文献[49]的方法合成, 中间体 SA-2 根据文献[50]合成, 所得数值与文献值一致.

3.2.1 2-(4-甲氧苯基)-4-甲基-1,3-硒唑-5-羧酸乙酯 (SA-3) 的合成

在室温条件下, 取 $0.4 \mathrm{~g}(0.0019 \mathrm{~mol})$ 的中间体 SA-2 于三颈瓶中, 加入 $15 \mathrm{~mL}$ 绝对无水乙醇搅拌溶解后, 滴 加 $0.265 \mathrm{~mL}(0.0019 \mathrm{~mol}) 2$-氯乙酰乙酸乙酯, 滴加完毕 后, 溶液为黄绿色, 搅拌 $15 \mathrm{~min}$, 转入油浴中, 温度缓 慢升至 $78{ }^{\circ} \mathrm{C}$, 回流, 用薄层色谱(TLC)监测反应, 反应 时间约为 $2 \mathrm{~h}$. 反应停止后, 趁热过滤, 冷却至室温, 滤 液为黄绿色, 倒入 $50 \mathrm{~mL}$ 冰水混合物中, 搅拌, 有浅粉 色固体析出, 抽滤, 固体用 $20 \mathrm{~mL}$ 乙醇重结晶得浅粉色 针状晶体 $0.505 \mathrm{~g}$, 收率 $82 \%$. m.p. 95.1 96.0 ${ }^{\circ} \mathrm{C} ;{ }^{1} \mathrm{H}$ NMR (500 MHz, DMSO- $\left.d_{6}\right) \delta: 7.86(\mathrm{~d}, J=8.80 \mathrm{~Hz}, 2 \mathrm{H}$, $\mathrm{PhH}), 6.94$ (d, $J=8.80 \mathrm{~Hz}, 2 \mathrm{H}, \mathrm{PhH}), 4.34$ (q, $J=7.10 \mathrm{~Hz}$, $\left.2 \mathrm{H}, \mathrm{CH}_{2}\right), 3.86\left(\mathrm{~s}, 3 \mathrm{H}, \mathrm{OCH}_{3}\right), 2.74\left(\mathrm{~s}, 3 \mathrm{H}, \mathrm{CH}_{3}\right), 1.38$ (t, $\left.J=7.15 \mathrm{~Hz}, 3 \mathrm{H}, \mathrm{CH}_{3}\right)$; IR (KBr) v: 3080, 2936, 1670, $1599,1465,1250,841 \mathrm{~cm}^{-1}$. HRMS (positive-ESIMS) calcd for $\mathrm{C}_{14} \mathrm{H}_{15} \mathrm{NO}_{3} \mathrm{Se}(\mathrm{M}+1)^{+}$325.0217, found 325.0226 .

\subsubsection{2-(4-甲氧苯基)-4-甲基-1,3-硒唑-5-碳酰肼(SA-} 4) 的合成

取 $0.972 \mathrm{~g}(0.003 \mathrm{~mol})$ 的中间体 SA-3 于三颈瓶中, 加入 $10 \mathrm{~mL}$ 乙醇摚拌溶解, 温度升至 $78{ }^{\circ} \mathrm{C}$, 缓慢滴加 7 $\mathrm{mL}$ 水合肼, 搅拌, 无色溶液逐渐变红, 回流, 溶液颜色
最后为红色，用 TLC 监测反应，反应时间约为 $14 \mathrm{~h}$. 反 应完成后趁热过滤, 滤液冷却至室温, 放入冰箱中冷冻 一夜，析出固体，抽滤得灰色固体，固体用 $20 \mathrm{~mL}$ 乙醇 重结晶得白色针状晶体 $0.558 \mathrm{~g}$, 收率 $60 \%$. m.p. 192.1 $193.2{ }^{\circ} \mathrm{C} ;{ }^{1} \mathrm{H}$ NMR (500 MHz, DMSO- $\left.d_{6}\right) \delta: 7.86(\mathrm{~d}, J=$ $8.80 \mathrm{~Hz}, 2 \mathrm{H}, \mathrm{PhH}), 6.94$ (d, J=8.80 Hz, 2H, PhH), 6.93 (s, $1 \mathrm{H}, \mathrm{NH}), 4.82\left(\mathrm{~s}, 2 \mathrm{H}, \mathrm{NH}_{2}\right), 3.86\left(\mathrm{~s}, 3 \mathrm{H}, \mathrm{OCH}_{3}\right), 2.74(\mathrm{~s}$, $\left.3 \mathrm{H}, \mathrm{CH}_{3}\right)$; IR (KBr) v: 3440, 3288, 3010, 2924, 1673, $1599,1500,1465,1240,845 \mathrm{~cm}^{-1}$. HRMS (positive-ESIMS) calcd for $\mathrm{C}_{12} \mathrm{H}_{13} \mathrm{~N}_{3} \mathrm{O}_{2} \mathrm{Se}(\mathrm{M}+1)^{+} 311.0173$, found 311.0182 .

3.2.3 2-(2-(4-甲氧苯基)-4-甲基-1,3-硒唑-5-羰基)- $N$ 苯基硫代氨基脲(SA-5)的合成

取 $0.31 \mathrm{~g}$ (0.001 mol) 中间体 SA-4 于三颈瓶中, 加入 $10 \mathrm{~mL}$ 乙醇, 温度升至 $78{ }^{\circ} \mathrm{C}$, 搅拌至完全溶解, 加入 $0.2 \mathrm{~mL}(0.015 \mathrm{~mol})$ 异硫氰酸苯酯, 搅拌, 体系为浅黄色 澄清溶液. 反应 $30 \mathrm{~min}$ 后, 黄色固体逐渐析出, 继续回 流反应, 用 TLC 监测反应, 反应时间约为 $8 \mathrm{~h}$. 反应完成 后, 静置冷却至室温, 将固体抽滤, 得粗产品并用乙醇 重结晶得浅黄色固体 $0.312 \mathrm{~g}$, 收率 70\%. m.p. 211.5 $212.3{ }^{\circ} \mathrm{C} ;{ }^{1} \mathrm{H}$ NMR (500 MHz, DMSO- $\left.d_{6}\right) \delta: 11.03$ (s, $1 \mathrm{H}$, $\mathrm{NH}), 10.16$ (s, 1H, NH), 9.81 (s, 1H, NH), 7.94 (d, J=8.75 $\mathrm{Hz}, 2 \mathrm{H}, \mathrm{PhH}), 7.35$ (t, $J=7.75 \mathrm{~Hz}, 3 \mathrm{H}, \mathrm{PhH}), 7.18$ (d, $J=$ $7.70 \mathrm{~Hz}, 2 \mathrm{H}, \mathrm{PhH}), 7.05$ (t, J=8.70 Hz, 2H, PhH), 3.85 (s, $\left.3 \mathrm{H}, \mathrm{OCH}_{3}\right), 2.65\left(\mathrm{~s}, 3 \mathrm{H}, \mathrm{CH}_{3}\right)$; IR (KBr) v: 3220, 3030, 2936, 1680, 1600, 1520, 1465, 1250, $845 \mathrm{~cm}^{-1}$. HRMS (positive-ESIMS) calcd for $\mathrm{C}_{19} \mathrm{H}_{18} \mathrm{~N}_{4} \mathrm{O}_{2} \mathrm{SSe}(\mathrm{M}+1)^{+}$ 446.0316, found 446.0322.

3.2.4 2-(4-甲氧苯基)-4-甲基-1,3-硒唑-硫钾盐(SA-6) 的合成

室温条件下, 取 $0.12 \mathrm{~g}(0.001 \mathrm{~mol}) \mathrm{KOH}$ 于三颈瓶 
中, 加入 $10 \mathrm{~mL}$ 乙醇, 搅拌至完全溶解后, 加入 $0.31 \mathrm{~g}$ (0.001 mol)中间体 SA-4, 搅拌, 缓慢滴加用 $2 \mathrm{~mL}$ 乙醇 稀释的 $0.2 \mathrm{~mL}(0.0015 \mathrm{~mol}) \mathrm{CS}_{2}$, 滴加完毕后, 体系为橘 黄色的澄清溶液, 搅拌 $10 \mathrm{~min}$ 后, 逐渐有黄色固体析出, 反应时间为 $10 \mathrm{~h}$. 将固体抽滤, 无水乙醇多次洗涤, 得 橘黄色固体 $0.369 \mathrm{~g}$, 收率: 87\%. m.p. $>300{ }^{\circ} \mathrm{C} ;{ }^{1} \mathrm{H}$ NMR $\left(500 \mathrm{MHz}, \mathrm{DMSO}-d_{6}\right) \delta: 10.95(\mathrm{~s}, 1 \mathrm{H}, \mathrm{NH}), 10.18(\mathrm{~s}, 1 \mathrm{H}$, $\mathrm{NH}), 11.07$ (s, 1H, SH), 7.65 (d, $J=8.60 \mathrm{~Hz}, 2 \mathrm{H}, \mathrm{PhH})$, 7.34 (d, $J=8.75 \mathrm{~Hz}, 2 \mathrm{H}, \mathrm{PhH}), 3.85$ (s, 3H, $\left.\mathrm{OCH}_{3}\right), 2.66$ (s, 3H, $\mathrm{CH}_{3}$ ); IR ( $\left.\mathrm{KBr}\right)$ v: 3240, 3030, 2936, 2560, 1675, $1610,1520,1460,1255,840 \mathrm{~cm}^{-1}$. HRMS (positive-ESIMS) calcd for $\mathrm{C}_{13} \mathrm{H}_{12} \mathrm{KN}_{3} \mathrm{O}_{2} \mathrm{~S}_{2} \mathrm{Se}(\mathrm{M}+1)^{+}$424.9173, found 424.9169 .

3.2.5 2-(2-(4-甲氧苯基)-4-甲基-1,3-硒唑-5-嶡基)硫 代氨基腺(SA-7)的合成

取 $0.31 \mathrm{~g}(0.001 \mathrm{~mol})$ 的中间体 $\mathbf{S A}-\mathbf{4}$ 于三颈瓶中, 加 入 $15 \mathrm{~mL} \mathrm{7 \%}$ 的稀盐酸, 摚拌, 加入 $0.19 \mathrm{~g}(0.002 \mathrm{~mol})$ $\mathrm{KSCN}$, 温度升至 $90{ }^{\circ} \mathrm{C}$, 搅拌至完全溶解, 反应 $4 \mathrm{~h}$ 后, 有固体析出, 用 TLC 监测反应. 反应停止后, 体系静置 冷却至室温, 抽滤, 得黄绿色固体, 用二氧六环重结晶 得浅黄色棒状晶体 $0.221 \mathrm{~g}$, 收率: $60 \%$. m.p. 287.5 $289.1{ }^{\circ} \mathrm{C} ;{ }^{1} \mathrm{H}$ NMR $\left(500 \mathrm{MHz}\right.$, DMSO- $\left.d_{6}\right) \delta: 10.65$ (s, $2 \mathrm{H}$, $\mathrm{NH}_{2}$ ), 10.16 (s, 1H, NH), 9.81 (s, 1H, NH), 7.94 (d, $J=$ $8.75 \mathrm{~Hz}, 2 \mathrm{H}, \mathrm{PhH}), 7.45$ (d, J=8.70 Hz, 2H, $\mathrm{PhH}$ ), 3.86 (s, $\left.3 \mathrm{H}, \mathrm{OCH}_{3}\right), 2.67\left(\mathrm{~s}, 3 \mathrm{H}, \mathrm{CH}_{3}\right)$; IR ( $\left.\mathrm{KBr}\right) v: 3420,3250$, 3030, 2936, 1680,1600,1520, 1465, 1253, $840 \mathrm{~cm}^{-1}$. HRMS (positive-ESIMS) calcd for $\mathrm{C}_{13} \mathrm{H}_{14} \mathrm{~N}_{4} \mathrm{O}_{2} \mathrm{SSe}(\mathrm{M}+$ 1) ${ }^{+} 370.0003$, found 370.0010 .

3.2.6 $\mathrm{N}^{\prime}$-(4-氯苯亚甲基)-2-(4-甲氧苯基)-4-甲基-1,3硒唑-5-碳酰胇(SA-8)的合成

取 $0.31 \mathrm{~g}(0.001 \mathrm{~mol})$ 中间体 $\mathbf{S A - 4}$ 于三颈瓶中, 加入 $10 \mathrm{~mL}$ 乙醇摚拌至完全溶解, 温度升至 $78{ }^{\circ} \mathrm{C}$, 再加入 $0.17 \mathrm{~g}(0.011 \mathrm{~mol})$ 对氯苯甲醛, 搅拌溶解, 反应 $4.5 \mathrm{~h}$ 后, 加入 $3 \sim 4$ 滴冰醋酸, 有黄绿色固体析出, 继续搅拌, 再 反应 $1 \mathrm{~h}$, 用 TLC 监测反应. 反应停止后, 将混合物倒入 $40 \mathrm{~mL}$ 冰水中, 搅拌, 有固体析出, 抽滤, 得黄色固体, 用二氧六环重结晶得黄色固体 $0.368 \mathrm{~g}$, 收率: $85 \%$. m.p. $245.7 \sim 247{ }^{\circ} \mathrm{C} ;{ }^{1} \mathrm{H}$ NMR (500 MHz, DMSO- $\left.d_{6}\right) \delta: 11.65$ (s, 1H, NH), 10.07 (s, 1H, CH), 7.86 (d, $J=8.60 \mathrm{~Hz}, 4 \mathrm{H}$, $\mathrm{PhH}), 7.04$ (d, $J=8.75 \mathrm{~Hz}, 4 \mathrm{H}, \mathrm{PhH}), 3.84$ (s, $3 \mathrm{H}, \mathrm{OCH}_{3}$ ), 2.76 (s, 3H, $\left.\mathrm{CH}_{3}\right)$; IR (KBr) v: 3260, 3030, 2936, 1673, $1603,1500,1465,1253,841,720 \mathrm{~cm}^{-1}$. HRMS (positiveESIMS) calcd for $\mathrm{C}_{19} \mathrm{H}_{16} \mathrm{ClN}_{3} \mathrm{O}_{2} \mathrm{Se}(\mathrm{M}+1)^{+}$433.0096, found 433.0103.
3.2.7 2-(4-甲氧苯基)-4-甲基- $N^{\prime}$-(丙烷-2-亚甲基)1,3-硒唑-5-碳酰胖(SA-9)的合成

取 $0.31 \mathrm{~g}(0.001 \mathrm{~mol})$ 中间体 $\mathbf{S A - 4}$ 于三颈瓶中, 加入 $10 \mathrm{~mL}$ 乙醇摚拌至完全溶解, 温度升至 $78{ }^{\circ} \mathrm{C}$, 加入 0.07 $\mathrm{mL}(0.0015 \mathrm{~mol})$ 丙酮，搅拌溶解回流，反应时间 $4 \mathrm{~h}$ 后， 加入 $3 \sim 4$ 滴冰醋酸, 继续摚拌, 再反应 $1 \mathrm{~h}$. 反应停止 后, 将混合物倒入 $40 \mathrm{~mL}$ 冰水中, 搅拌, 有黄色固体析 出, 用二氧六环重结晶得黄色固体 $0.298 \mathrm{~g}$, 收率: 85.2\%. m.p. $223.2 \sim 224.8{ }^{\circ} \mathrm{C} ;{ }^{1} \mathrm{H}$ NMR $(500 \mathrm{MHz}$, DMSO- $\left.d_{6}\right) \delta: 11.65(\mathrm{~s}, 1 \mathrm{H}, \mathrm{NH}), 7.90 \sim 7.88(\mathrm{~m}, 2 \mathrm{H}, \mathrm{PhH})$, $7.05 \sim 7.02(\mathrm{~m}, 2 \mathrm{H}, \mathrm{PhH}), 3.84\left(\mathrm{~s}, 3 \mathrm{H}, \mathrm{OCH}_{3}\right), 2.72(\mathrm{~s}, 3 \mathrm{H}$, $\left.\mathrm{CH}_{3}\right), 1.79$ (s, 6H, $\mathrm{CH}_{3}$ ); IR ( $\left.\mathrm{KBr}\right)$ v: 3255, 3030, 2930, $1670,1600,1500,1465,1391,1253,845 \mathrm{~cm}^{-1}$. HRMS (positive-ESIMS) calcd for $\mathrm{C}_{15} \mathrm{H}_{17} \mathrm{~N}_{3} \mathrm{O}_{2} \mathrm{Se}(\mathrm{M}+1)^{+}$ 351.0486, found 351.0478 .

3.2.8 2-(4-甲氧苯基)-4-甲基-1,3-硒唑-甲酰胺(SA10)的合成

在冰浴条件下，向 $0.64 \mathrm{~g}(0.002 \mathrm{~mol})$ 的中间体 SA-3 的甲醇浊液中通入氨气, 搅拌, 固体逐渐溶解, 体系为 黄色澄清溶液, 继续通氨气, 时间约为 $6 \mathrm{~h}$ 后, 转移至室 温, 继续摚拌反应 $24 \mathrm{~h}$. 反应停止后, 将氨气减压抽出,

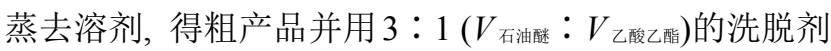
进行柱层析得白色固体 $0.295 \mathrm{~g}$, 收率: $50 \%$. m.p. $245.7 \sim 247.0{ }^{\circ} \mathrm{C} ;{ }^{1} \mathrm{H}$ NMR (500 MHz, DMSO- $\left.d_{6}\right) \delta: 7.86$ (d, $J=8.80 \mathrm{~Hz}, 2 \mathrm{H}, \mathrm{Ph}), 6.94(\mathrm{~d}, J=8.80 \mathrm{~Hz}, 2 \mathrm{H}, \mathrm{Ph})$, 4.87 (s, $\left.2 \mathrm{H}, \mathrm{NH}_{2}\right), 3.84$ (s, 3H, $\left.\mathrm{OCH}_{3}\right), 2.72\left(\mathrm{~s}, 3 \mathrm{H}, \mathrm{CH}_{3}\right)$; IR (KBr) v: 3430, 3033, 2930, 1670, 1606, 1505, 1460, $1260,845 \mathrm{~cm}^{-1}$. HRMS (positive-ESIMS) calcd for $\mathrm{C}_{12} \mathrm{H}_{12} \mathrm{~N}_{2} \mathrm{O}_{2} \mathrm{Se}(\mathrm{M}+1)^{+} 296.0064$, found 296.0070.

3.2.92-(4-甲氧苯基)-4-甲基-1,3-硒唑-甲腈(SA-11) 的合成

取 $0.296 \mathrm{~g}(0.001 \mathrm{~mol})$ 中间体 SA-10 于三颈瓶中, 加 入 $10 \mathrm{~mL}$ 乙腈, 搅拌, 温度升至 $80{ }^{\circ} \mathrm{C}$, 加入 $3 \mathrm{~mL}$ 干燥 的三氯氧磷，摚拌反应约为 $5 \mathrm{~h}$. 反应停止后, 混合物冷 却至室温, 加入冰水, 搅拌, 固体析出, 抽滤, 干燥得白 色固体 $0.175 \mathrm{~g}$, 收率: $60 \%$. m.p. $145.8 \sim 147.2{ }^{\circ} \mathrm{C} ;{ }^{1} \mathrm{H}$ NMR $\left(500 \mathrm{MHz}\right.$, DMSO- $\left.d_{6}\right) \delta: 7.84(\mathrm{~d}, J=8.80 \mathrm{~Hz}, 2 \mathrm{H}$, $\mathrm{PhH}$ ), 7.05 (d, $J=8.80 \mathrm{~Hz}, 2 \mathrm{H}, \mathrm{PhH}), 3.86\left(\mathrm{~s}, 3 \mathrm{H}, \mathrm{OCH}_{3}\right.$ ), 2.74 (s, 3H, $\mathrm{CH}_{3}$ ); IR (KBr) v: 3033, 2934, 2350, 1600, $1500,1450,1250,845 \mathrm{~cm}^{-1}$. HRMS (positive-ESIMS) calcd for $\mathrm{C}_{12} \mathrm{H}_{10} \mathrm{~N}_{2} \mathrm{OSe}(\mathrm{M}+1)^{+}$277.9958, found 277.9965 .

3.2.10 3-(2-(2-(4-甲氧苯基)-4-甲基-1,3-硒唑-5-羰 基)肼)-3-甲酰乙酸甲酯(SA-12)的合成

取 $70 \mu \mathrm{L}(0.0015 \mathrm{~mol})$ 丙二酸二甲酯和 $8 \mathrm{~mL}$ THF 于 
三颈瓶中, 充分搅拌, 温度升至 $66{ }^{\circ} \mathrm{C}$, 加入 $80 \mu \mathrm{L}$ $(0.0015 \mathrm{~mol})$ 三乙胺, 搅拌 $30 \mathrm{~min}$ 后, 加入 $0.31 \mathrm{~g}(0.001$ mol)中间体 SA-4, 搅拌完全溶解, 体系为黄色溶液, 回 流, 用 TLC 监测反应, 反应时间约为 $5 \mathrm{~h}$. 反应停止后, 静置冷却至室温, 将溶剂减压蒸去, 得橘黄色固体 0.287 g, 收率: $70 \%$. m.p. $276.8 \sim 277.9{ }^{\circ} \mathrm{C}$; ${ }^{1} \mathrm{H}$ NMR $\left(500 \mathrm{MHz}, \mathrm{DMSO}-d_{6}\right) \delta: 10.12(\mathrm{~s}, 1 \mathrm{H}, \mathrm{NH}), 9.80(\mathrm{~s}, 1 \mathrm{H}$, $\mathrm{NH}), 7.72(\mathrm{~d}, J=8.75 \mathrm{~Hz}, 2 \mathrm{H}, \mathrm{PhH}), 7.05(\mathrm{t}, J=8.70 \mathrm{~Hz}$, $2 \mathrm{H}, \mathrm{PhH}), 3.85$ (s, $\left.3 \mathrm{H}, \mathrm{OCH}_{3}\right), 3.64\left(\mathrm{~s}, 3 \mathrm{H}, \mathrm{CH}_{3}\right), 3.50$ (s, $2 \mathrm{H}, \mathrm{CH}_{2}$ ), 2.65 (s, 3H, $\mathrm{CH}_{3}$ ); IR ( $\left.\mathrm{KBr}\right)$ v: 3267, 3030, 2940, 1680, 1600,1500, 1455, 1390, 1250, 850, $730 \mathrm{~cm}^{-1}$. HRMS (positive-ESIMS) calcd for $\mathrm{C}_{16} \mathrm{H}_{17} \mathrm{~N}_{3} \mathrm{O}_{5} \mathrm{Se}(\mathrm{M}+1)^{+}$ 411.0333, found 411.0339.

\section{3 化合物 SAZ-1 SAZ-22 的合成}

3.3.1 (3,5-二甲基- $1 H$-吡唑-1-基)-(2-(4-甲氧苯基)-4甲基-1,3-硒唑-5-基)甲酮(SAZ-1)的合成

向三颈瓶中加入 $0.31 \mathrm{~g}(0.001 \mathrm{~mol})$ 的中间体 SA-4 和 $8 \mathrm{~mL}$ 乙醇, 搅拌, 温度升至 $78{ }^{\circ} \mathrm{C}$, 完全溶解后, 加 入 $57 \mu \mathrm{L}(0.0012 \mathrm{~mol})$ 乙酰丙酮, 搅拌回流, 用 TLC 监测 反应, 反应时间约为 $10 \mathrm{~h}$. 反应停止后, 静置冷却至室 温, 将混合物倒入冰水中, 搅拌, 固体析出, 抽滤, 固体 粗产品用无水乙醇重结晶得黄色针状晶体 $0.319 \mathrm{~g}$, 收 率为 $85 \%$. m.p. $166.8 \sim 168.0{ }^{\circ} \mathrm{C} ;{ }^{1} \mathrm{H}$ NMR $(500 \mathrm{MHz}$, DMSO- $\left.d_{6}\right) \delta: 8.00(\mathrm{~d}, J=8.65 \mathrm{~Hz}, 2 \mathrm{H}, \mathrm{PhH}), 6.96(\mathrm{~d}, J=$ $8.65 \mathrm{~Hz}, 2 \mathrm{H}, \mathrm{PhH}), 6.04(\mathrm{~s}, 1 \mathrm{H}, \mathrm{CH}=\mathrm{C}), 3.87(\mathrm{~s}, 3 \mathrm{H}$, $\left.\mathrm{OCH}_{3}\right), 2.91$ (s, $\left.3 \mathrm{H}, \mathrm{CH}_{3}\right), 2.65$ (s, $\left.3 \mathrm{H}, \mathrm{CH}_{3}\right), 2.30(\mathrm{~s}, 3 \mathrm{H}$, $\mathrm{CH}_{3}$ ); ${ }^{13} \mathrm{C}$ NMR $\left(125 \mathrm{MHz}, \mathrm{DMSO}-d_{6}\right.$ ) $\delta: 182.95$ (硒唑 环), $166.07(\mathrm{C}=\mathrm{O}), 162.53$ (硒唑环), 162.30 (硒唑环), 151.35 (吡唑环), 144.80 (吡唑环), $129.10(\mathrm{Ph}), 128.71$ $(\mathrm{Ph}), 115.22(\mathrm{Ph}), 112.39$ (吡唑环), $55.99\left(\mathrm{OCH}_{3}\right), 21.26$ $\left(\mathrm{CH}_{3}\right), 14.93\left(\mathrm{CH}_{3}\right), 13.85\left(\mathrm{CH}_{3}\right)$; IR ( $\left.\mathrm{KBr}\right)$ v: 3030, 2960 , 2840, 1665, 1606, 1520, 1483, 1250, $828 \mathrm{~cm}^{-1}$; HRMS (positive-ESIMS) calcd for $\mathrm{C}_{17} \mathrm{H}_{17} \mathrm{~N}_{3} \mathrm{O}_{2} \mathrm{Se}(\mathrm{M}+1)^{+}$ 375.0486, found 375.0496 .

3.3.2 1-(2-(4-甲氧苯基)-4-甲基-1,3-硒唑-5-羰基)-3甲基- $1 H$-吡唑-5(4H)-酮(SAZ-2)的合成

向三颈瓶中加入 $0.31 \mathrm{~g}(0.001 \mathrm{~mol})$ 中间体 SA-4 和 8 $\mathrm{mL}$ 乙醇, 搅拌, 温度升至 $78{ }^{\circ} \mathrm{C}$, 完全溶解后, 加入 63 $\mu \mathrm{L}(0.0012 \mathrm{~mol})$ 乙酰乙酸乙酯, 搅拌, 加入 $0.1 \mathrm{~g}(0.0012$ mol) $\mathrm{K}_{2} \mathrm{CO}_{3}$ 回流, 用 TLC 监测反应, 反应时间约为 14 h. 反应停止后, 静置冷却至室温, 将混合物倒入冰水 中, 搅拌, 固体析出, 抽滤, 用乙醇对固体进行重结晶 得浅黄色针状晶体 $0.283 \mathrm{~g}$, 收率为 $75 \%$. m.p. 172.5 $174.0{ }^{\circ} \mathrm{C} ;{ }^{1} \mathrm{H}$ NMR $\left(500 \mathrm{MHz}\right.$, DMSO- $\left.d_{6}\right) \delta$ : $7.94(\mathrm{~d}, J=$ $8.70 \mathrm{~Hz}, 2 \mathrm{H}, \mathrm{PhH}), 7.04$ (q, $J=8.70 \mathrm{~Hz}, 2 \mathrm{H}, \mathrm{PhH}), 4.14$ (d, $\left.J=7.10 \mathrm{~Hz}, 2 \mathrm{H}, \mathrm{CH}_{2}\right), 3.83$ (s, 3H, $\left.\mathrm{OCH}_{3}\right), 2.73$ (s, $3 \mathrm{H}$, $\left.\mathrm{CH}_{3}\right), 2.07\left(\mathrm{~s}, 3 \mathrm{H}, \mathrm{CH}_{3}\right) ;{ }^{13} \mathrm{C}$ NMR (125 MHz, DMSO- $\left.d_{6}\right)$ $\delta: 182.90$ (硒唑环), $166.07(\mathrm{C}=\mathrm{O}), 162.54$ (硒唑环), 162.31 (硒唑), 154.46 (吡唑环), 151.34 (吡唑环), 144.80 (吡唑环), $129.10(\mathrm{Ph}), 128.71(\mathrm{Ph}), 115.22(\mathrm{Ph}), 112.38$ $(\mathrm{Ph}), 55.99\left(\mathrm{OCH}_{3}\right), 21.24\left(\mathrm{CH}_{3}\right), 14.90\left(\mathrm{CH}_{3}\right) ; \mathrm{IR}(\mathrm{KBr}) v$ : $3047,1723,1636,1432,1257,830 \mathrm{~cm}^{-1}$; HRMS (positiveESIMS) calcd for $\mathrm{C}_{16} \mathrm{H}_{15} \mathrm{~N}_{3} \mathrm{O}_{3} \mathrm{Se}(\mathrm{M}+1)^{+} 377.0279$, found 377.0289 .

3.3.3 4-氨基-5-(2-(4-甲氧苯基)-4-甲基-1,3-硒唑- 5基)-4H-1,2,4-三唑-3-硫醇(SAZ-3)的合成

取 $0.5 \mathrm{~g}(0.0015 \mathrm{~mol})$ 中间体 $\mathbf{S A - 6}$ 完全溶解于 $8 \mathrm{~mL}$ 水合肼中, 升温至 $90{ }^{\circ} \mathrm{C}$, 摚拌, 用 TLC 监测反应, 反应 时间为 $6 \mathrm{~h}$. 将混合物倒入 $50 \mathrm{~mL}$ 冰水混合物中搅拌, 冰 完全溶解后, 加入稀盐酸调节 PH 值约为 $3 \sim 4$, 有橘红 色固体析出, 抽滤, 固体粗产品用乙醇重结晶得橘红色 棒状晶体 $0.347 \mathrm{~g}$, 收率为 $63 \%{ }^{[51]}$. m.p. $256.8 \sim 257.9{ }^{\circ} \mathrm{C}$; ${ }^{1} \mathrm{H}$ NMR (500 MHz, DMSO- $d_{6}$ ) $\delta: 13.98$ (s, 1H, SH), 7.94 (d, $J=8.70 \mathrm{~Hz}, 2 \mathrm{H}, \mathrm{PhH}), 7.05(\mathrm{~d}, J=8.70 \mathrm{~Hz}, 2 \mathrm{H}, \mathrm{PhH}$ ), $5.90\left(\mathrm{~s}, 2 \mathrm{H}, \mathrm{NH}_{2}\right), 3.83\left(\mathrm{~d}, J=3.45 \mathrm{~Hz}, 3 \mathrm{H}, \mathrm{OCH}_{3}\right), 2.69$ (s, $\left.3 \mathrm{H}, \mathrm{CH}_{3}\right) ;{ }^{13} \mathrm{C}$ NMR (125 MHz, DMSO- $\left.d_{6}\right) \delta: 173.50$ (硒唑环), 162.33 (硒唑环), 160.33 (硒唑环), 154.60 (三 唑环), 146.63 (三号环), $129.16(\mathrm{Ph}), 128.94(\mathrm{Ph}), 128.40$ $(\mathrm{Ph}), 127.18(\mathrm{Ph}), 119.57(\mathrm{Ph}), 115.14(\mathrm{Ph}), 55.92\left(\mathrm{OCH}_{3}\right)$, $18.20\left(\mathrm{CH}_{3}\right)$; IR (KBr) v: 3424, 3106, 2924, 1650, 1600, 1250, $845 \mathrm{~cm}^{-1}$; HRMS (positive-ESIMS) calcd for $\mathrm{C}_{13} \mathrm{H}_{13} \mathrm{~N}_{5} \mathrm{OSSe}(\mathrm{M}+1)^{+} 367.0006$, found 367.0015 .

3.3.4 5-(2-(4-甲氧苯基)-4-甲基-1,3-硒唑-5-基)-4-苯 基-4H-1,2,4-三唑-3-硫醇(SAZ-4)的合成

取 $0.478 \mathrm{~g}(0.001 \mathrm{~mol})$ 的中间体 SA-5 于三颈瓶中, 加入 $15 \mathrm{~mL}$ 的 $10 \%$ 的 $\mathrm{NaOH}$ 水溶液, 升温至 $90{ }^{\circ} \mathrm{C}$, 摚 拌, 固体逐渐溶解完全, 溶液为黄色. 用 TLC 监测反应, 反应时间约为 $7 \mathrm{~h}$. 趁热过滤, 滤液冷却至室温, 倒入 50 $\mathrm{mL}$ 冰水混合物中, 冰完全融化后, 加入 $2 \mathrm{~mol} / \mathrm{L}$ 的稀盐 酸调节 PH 值约为 6 , 有淡黄色固体析出, 抽滤, 用无水 乙醇重结晶得淡黄色针状晶体 $0.299 \mathrm{~g}$, 收率为 $70 \%$. m.p. 206.8 208.2 ${ }^{\circ} \mathrm{C} ;{ }^{1} \mathrm{H}$ NMR (500 MHz, DMSO- $\left.d_{6}\right) \delta$ : 14.22 (s, 1H, SH), 7.72 (q, $J=6.85 \mathrm{~Hz}, 2 \mathrm{H}, \mathrm{PhH}), 7.53$ (t, $J=6.85 \mathrm{~Hz}, 3 \mathrm{H}, \mathrm{PhH}), 7.43 \sim 7.41(\mathrm{~m}, 2 \mathrm{H}, \mathrm{PhH}), 6.98(\mathrm{q}$, $J=6.85 \mathrm{~Hz}, 2 \mathrm{H}, \mathrm{PhH}), 3.80$ (s, 3H, $\left.\mathrm{OCH}_{3}\right), 2.41$ (s, $3 \mathrm{H}$, $\left.\mathrm{CH}_{3}\right) ;{ }^{13} \mathrm{C}$ NMR (125 MHz, DMSO- $d_{6}$ ) $\delta: 175.23$ (硒唑环), 168.55 (硒唑环), 162.07 (硒唑环), 156.42 (三唑环), 147.14 (三唑环), $134.18(\mathrm{Ph}), 130.45(\mathrm{Ph}), 129.91(\mathrm{Ph})$, $129.59(\mathrm{Ph}), 128.81(\mathrm{Ph}), 128.13(\mathrm{Ph}), 118.50(\mathrm{Ph}), 115.16$ $(\mathrm{Ph}), 55.93\left(\mathrm{OCH}_{3}\right), 18.20\left(\mathrm{CH}_{3}\right)$; IR (KBr) v: 3069, 2931, 
$1606,1512,1453,1250,828 \mathrm{~cm}^{-1}$; HRMS (positive-ESIMS) calcd for $\mathrm{C}_{19} \mathrm{H}_{16} \mathrm{~N}_{4} \mathrm{OSSe}(\mathrm{M}+1)^{+} 428.0210$, found 428.0200 .

3.3.5 3-(2-(4-甲氧苯基)-4-甲基-1,3-硒唑-5-基)- $1 H$ 1,2,4-三唑-5-硫醇(SAZ-5)的合成

取 $0.37 \mathrm{~g}(0.001 \mathrm{~mol})$ 的中间体 SA-7 于三颈瓶中, 加 入 $20 \mathrm{~mL}$ (20 equiv.) $5 \% \mathrm{NaOH}$ 水溶液, 搅拌, 温度升至 $90{ }^{\circ} \mathrm{C}$, 体系为澄清黄色溶液, 用 TLC 监测反应, 反应 时间为 $6.5 \mathrm{~h}$. 反应停止后, 趁热过滤, 滤液冷却至室温, 用 $2 \mathrm{~mol} / \mathrm{L}$ 的稀盐酸溶液调节 $\mathrm{PH}$ 值为 5 , 搅拌, 有黄色 固体析出, 抽滤并用乙醇重结晶得黄色固体 $0.217 \mathrm{~g}$, 收 率为 61.7\%. m.p. $252.6 \sim 254.1{ }^{\circ} \mathrm{C} ;{ }^{1} \mathrm{H}$ NMR $(500 \mathrm{MHz}$, DMSO- $\left.d_{6}\right) \delta: 13.76(\mathrm{~s}, 1 \mathrm{H}, \mathrm{SH}), 13.71(\mathrm{~s}, 1 \mathrm{H}, \mathrm{NH}), 7.90(\mathrm{t}$, $J=8.70 \mathrm{~Hz}, 2 \mathrm{H}, \mathrm{PhH}), 7.05 \sim 7.02(\mathrm{~m}, 2 \mathrm{H}, \mathrm{PhH}), 3.84(\mathrm{~s}$, $\left.3 \mathrm{H}, \mathrm{OCH}_{3}\right), 2.71\left(\mathrm{~s}, 3 \mathrm{H}, \mathrm{CH}_{3}\right) ;{ }^{13} \mathrm{C}$ NMR $(125 \mathrm{MHz}$, DMSO- $\left.d_{6}\right) \delta: 173.57$ (硒唑环), 162.32 (硒夾环), 160.33 (硒唑环), 154.64 (三唑环), 146.63 (三唑环), $129.15(\mathrm{Ph})$, $128.59(\mathrm{Ph}), 128.41(\mathrm{Ph}), 127.18(\mathrm{Ph}), 119.57(\mathrm{Ph}), 115.14$ $(\mathrm{Ph}), 55.94\left(\mathrm{OCH}_{3}\right), 18.33\left(\mathrm{CH}_{3}\right)$; IR $(\mathrm{KBr}) v: 3290,3069$, 2931, 1640, 1600, 1512, 1453, 1250, $828 \mathrm{~cm}^{-1}$; HRMS (positive-ESIMS) calcd for $\mathrm{C}_{13} \mathrm{H}_{12} \mathrm{~N}_{4} \mathrm{OSSe}(\mathrm{M}+1)^{+}$ 351.9897, found 351.9887.

3.3.6 2-(2-(4-甲氧苯基)-4-甲基-1,3-硒唑-5-基)1,3,4-愳二唑(SAZ-6) 的合成

取 $0.31 \mathrm{~g}(0.001 \mathrm{~mol})$ 中间体 $\mathbf{S A - 4}$ 于三颈瓶中, 加入 $8 \mathrm{~mL}$ 原甲酸三乙酯, 搅拌, 温度升至 $90{ }^{\circ} \mathrm{C}$, 固体全部 溶解, 体系为无色澄清溶液, 用 TLC 监测反应, 反应时 间为 $14 \mathrm{~h}$. 反应停止后, 静置冷却至室温, 放入冰箱中 冷冻一夜, 有灰色固体析出, 抽滤, 固体用无水乙醇重 结晶, 得灰白色针状晶体 $0.234 \mathrm{~g}$, 收率为 $73 \%$. m.p. $247.8 \sim 249.1{ }^{\circ} \mathrm{C} ;{ }^{1} \mathrm{H}$ NMR (500 MHz, DMSO- $\left.d_{6}\right) \delta$ : 10.89 (s, 1H, CH), $8.07 \sim 7.89$ (m, 2H, PhH), $7.08 \sim 6.99$ $(\mathrm{m}, 2 \mathrm{H}, \mathrm{PhH}), 3.84\left(\mathrm{~d}, J=9.35 \mathrm{~Hz}, 3 \mathrm{H}, \mathrm{OCH}_{3}\right), 2.72(\mathrm{~s}$, $\left.3 \mathrm{H}, \mathrm{CH}_{3}\right) ;{ }^{13} \mathrm{C}$ NMR (125 MHz, DMSO- $d_{6}$ ) $\delta: 175.05$ (硒 唑环), 162.45 (硒唑环), 161.23 (硒唑环), 154.46 (噁二唑 环), 150.18 (啞二唑环), $129.32(\mathrm{Ph}), 128.84(\mathrm{Ph}), 128.77$ $(\mathrm{Ph}), 128.13(\mathrm{Ph}), 115.25(\mathrm{Ph}), 115.13(\mathrm{Ph}), 56.01\left(\mathrm{OCH}_{3}\right)$, $18.37\left(\mathrm{CH}_{3}\right)$; IR (KBr) v: 3047, 1640, 1613, 1498, 1250, $842,755 \mathrm{~cm}^{-1}$; HRMS (positive-ESIMS) calcd for $\mathrm{C}_{13} \mathrm{H}_{11^{-}}$ $\mathrm{N}_{3} \mathrm{O}_{2} \mathrm{Se}(\mathrm{M}+1)^{+} 321.0016$, found 321.0010 .

3.3.75-(2-(4-甲氧苯基)-4-甲基-1,3-硒唑-5-基)1,3,4-哣二唑-2(3H)-酮(SAZ-7) 的合成

向三颈瓶中加入 $0.31 \mathrm{~g}(0.001 \mathrm{~mol})$ 的中间体 SA-4 和 $8 \mathrm{~mL}$ 二氧六环, 搅拌至完全溶解, 体系为黄色澄清 溶液, 加入白色粉末状的 $N, N$-羰基二咪唑(CDI), 摚拌
完全溶解，回流，TLC 监测反应，反应时间约为 $4 \mathrm{~h}$. 反 应停止后, 静置冷却至室温, 减压蒸去溶剂, 得黄色固 体. 向固体中加入 $30 \mathrm{~mL}$ 水, 充分搅拌, 抽滤, 固体干 燥得黄色固体 $0.286 \mathrm{~g}$, 收率为 $85 \%$. m.p. 218.5 $219.2{ }^{\circ} \mathrm{C} ;{ }^{1} \mathrm{H}$ NMR $\left(500 \mathrm{MHz}\right.$, DMSO- $\left.d_{6}\right) \delta: 12.69$ (s, $1 \mathrm{H}$, $\mathrm{NH}), 7.96$ (d, $J=8.75 \mathrm{~Hz}, 2 \mathrm{H}, \mathrm{PhH}), 7.11(\mathrm{~d}, J=8.75 \mathrm{~Hz}$, $2 \mathrm{H}, \mathrm{PhH}), 3.89\left(\mathrm{~s}, 3 \mathrm{H}, \mathrm{OCH}_{3}\right), 2.66\left(\mathrm{~s}, 3 \mathrm{H}, \mathrm{CH}_{3}\right) ;{ }^{13} \mathrm{C}$ NMR (125 MHz, DMSO- $d_{6}$ ) $\delta: 173.64$ (硒唑环), 162.29 (硒唑环), 162.19 (硒唑环), 155.12 (噁二唑环), 151.67 (噁二唑环), $129.17(\mathrm{Ph}), 129.10(\mathrm{Ph}), 128.18(\mathrm{Ph}), 128.14$ $(\mathrm{Ph}), 118.43(\mathrm{Ph}), 115.21(\mathrm{Ph}), 55.99\left(\mathrm{OCH}_{3}\right), 18.24$ $\left(\mathrm{CH}_{3}\right)$; IR (KBr) v: 3244, 3011, 1796, 1600, 1447, 1257, $820 \mathrm{~cm}^{-1}$; HRMS (positive-ESIMS) calcd for $\mathrm{C}_{13} \mathrm{H}_{11} \mathrm{~N}_{3} \mathrm{O}_{3}$ Se $(\mathrm{M}+1)^{+}$336.9966, found 336.9950 .

3.3.8 5-(2-(4-甲氧苯基)-4-甲基-1,3-硒唑-5-基)-1,3， 4-噁二唑-2-硫醇(SAZ-8) 的合成

在室温条件下, 取 $0.028 \mathrm{~g}(0.0015 \mathrm{~mol}) \mathrm{KOH}$ 于三 口瓶中, 加入 $10 \mathrm{~mL}$ 乙醇, 搅拌至完全溶解, 加入中间 体 $0.31 \mathrm{~g}(0.001 \mathrm{~mol}) \mathbf{S A - 4}$, 搅拌至完全溶解后, 再缓慢 滴加 $0.2 \mathrm{~mL}(0.0015 \mathrm{~mol}) \mathrm{CS}_{2}$, 体系由黄色变为橘黄色 溶液, 滴加完毕后, 继续搅拌 $3 \mathrm{~min}$, 逐渐有黄色固体析 出, 继续搅拌 $4 \mathrm{~h}$. 将体系转移至油浴中, 加热回流约 5 $\mathrm{h}$. 反应停止后, 将混合物倒入冰水中, 搅拌, 为黄色澄 清溶液, 加稀盐酸调节 PH 约为 $4 \sim 5$, 有黄色固体析出, 抽滤，用乙醇对固体进行重结晶得黄色棒状晶体 0.268 $\mathrm{g}$, 收率为 $76.1 \%{ }^{[52]}$. m.p. $268.3 \sim 269.6{ }^{\circ} \mathrm{C} ;{ }^{1} \mathrm{H}$ NMR $(500$ MHz, DMSO- $\left.d_{6}\right) \delta: 14.80(\mathrm{~s}, 1 \mathrm{H}, \mathrm{SH}), 7.94(\mathrm{~d}, J=8.70$ $\mathrm{Hz}, 2 \mathrm{H}, \mathrm{PhH}), 7.06$ (d, J=8.70 Hz, 2H, PhH), 3.84 (s, 3H, $\left.\mathrm{OCH}_{3}\right), 2.66\left(\mathrm{~s}, 3 \mathrm{H}, \mathrm{CH}_{3}\right) ;{ }^{13} \mathrm{C}$ NMR $(125 \mathrm{MHz}$, DMSO$\left.d_{6}\right) \delta: 177.21$ (硒唑环), 174.99 (硒唑环), 162.46 (硒唑环), 158.21 (噁二唑环), 157.00 (噁二唑环), $129.28(\mathrm{Ph})$, $127.978(\mathrm{Ph}), 116.34(\mathrm{Ph}), 115.17(\mathrm{Ph}), 55.99\left(\mathrm{OCH}_{3}\right)$, $18.42\left(\mathrm{CH}_{3}\right)$; IR (KBr) v: 3034, 2917, 1640, 1610, 1500, 1450, 1373, 1250, $845 \mathrm{~cm}^{-1}$; HRMS (positive-ESIMS) calcd for $\mathrm{C}_{13} \mathrm{H}_{11} \mathrm{~N}_{3} \mathrm{O}_{2} \mathrm{SSe}(\mathrm{M}+1)^{+}$352.9737, found 352.9730 .

3.3.9 5-(2-(4-甲氧苯基)-4-甲基-1,3-硒唑-5-基)- $N$-苯 基-1,3,4-吘二唑-2-胺(SAZ-9)的合成

向三颈瓶中加入 $0.478 \mathrm{~g}(0.001 \mathrm{~mol})$ 中间体 SA-5, 加入 $8 \mathrm{~mL}$ 吡啶, 搅拌, 体系为黄色溶液, 回流, 用 TLC 监测反应, 反应时间约为 $8 \mathrm{~h}$. 反应停止, 静置冷却至室 温, 将混合物倒入冰水中, 摚拌, 有黄色固体析出, 抽 滤, 固体粗产品用无水乙醇重结晶的黄色棒状晶体 $0.314 \mathrm{~g}$, 收率为 $76.1 \%$. m.p. $265.9 \sim 267.1{ }^{\circ} \mathrm{C} ;{ }^{1} \mathrm{H}$ NMR $\left(500 \mathrm{MHz}, \mathrm{DMSO}-d_{6}\right) \delta: 10.68(\mathrm{~s}, 1 \mathrm{H}, \mathrm{NH}), 7.93(\mathrm{~d}, J=$ 
$8.45 \mathrm{~Hz}, 2 \mathrm{H}, \mathrm{PhH}), 7.61$ (d, J=7.80 Hz, 2H, PhH), 7.38 (t, $J=7.80 \mathrm{~Hz}, 2 \mathrm{H}, \mathrm{PhH}), 7.07$ (q, $J=8.45 \mathrm{~Hz}, 3 \mathrm{H}, \mathrm{PhH}$ ), $3.84\left(\mathrm{~s}, 3 \mathrm{H}, \mathrm{OCH}_{3}\right), 2.71\left(\mathrm{~s}, 3 \mathrm{H}, \mathrm{CH}_{3}\right) ;{ }^{13} \mathrm{C}$ NMR $(125$ MHz, DMSO- $\left.d_{6}\right) \delta: 173.28$ (硒唑环), 162.23 (硒唑环), 159.95 (硒唑环), 155.21 (噁二唑环), 154.78 (噁二唑环), $138.95(\mathrm{Ph}), 129.57(\mathrm{Ph}), 129.15(\mathrm{Ph}), 128.28(\mathrm{Ph}), 122.46$ $(\mathrm{Ph}), 117.81(\mathrm{Ph}), 117.59(\mathrm{Ph}), 115.20(\mathrm{Ph}), 55.99\left(\mathrm{OCH}_{3}\right)$, $18.26\left(\mathrm{CH}_{3}\right)$; IR (KBr) v: 3270, 3040, 1641, 1616, 1498, 1440, 1250, 747, $682 \mathrm{~cm}^{-1}$; HRMS (positive-ESIMS) calcd for $\mathrm{C}_{19} \mathrm{H}_{16} \mathrm{~N}_{4} \mathrm{O}_{2} \mathrm{Se}(\mathrm{M}+1)^{+} 412.0438$, found 412.0430 .

3.3.10 5-(2-(4-甲氧苯基)-4-甲基-1,3-硒唑-5-基)-2,2二甲基-1,3,4-噁二唑-3(2H)-基)-乙烯酮(SAZ-10)的合 成

取 $0.35 \mathrm{~g}(0.001 \mathrm{~mol})$ 中间体 $\mathbf{S A - 9}$ 于三颈瓶中, 加入 $8 \mathrm{~mL}$ 乙酸酲, 摚拌至完全溶解, 回流, 用 TLC 监测反 应, 反应时间约为 $4 \mathrm{~h}$. 反应停止后, 冷却至室温, 将混 合物倒入冰水混合物中, 搅拌, 有黄色固体析出, 抽滤, 干燥, 得黄色固体 $0.239 \mathrm{~g}$, 收率为 $63 \%$. m.p. 251.2 $252.9{ }^{\circ} \mathrm{C}$; ${ }^{1} \mathrm{H}$ NMR $\left(500 \mathrm{MHz}\right.$, DMSO- $\left.d_{6}\right) \delta: 7.90 \sim 7.88$ (m, 2H, PhH), $7.06 \sim 7.03$ (m, 2H, PhH), 3.84 (d, $J=6.60$ $\left.\mathrm{Hz}, 3 \mathrm{H}, \mathrm{OCH}_{3}\right), 2.71$ (s, $\left.3 \mathrm{H}, \mathrm{CH}_{3}\right), 2.18$ (s, 3H, $\left.\mathrm{CH}_{3}\right), 1.79$ (s, 6H, $\left.\mathrm{CH}_{3}\right) ;{ }^{13} \mathrm{C}$ NMR (125 MHz, DMSO- $\left.d_{6}\right) \delta: 173.97$ (硒唑环), $166.18(\mathrm{C}=\mathrm{O}), 162.30$ (硒唑环), 156.77 (硒唑 环), 150.76 (噁二唑环), $129.15(\mathrm{Ph}), 128.61(\mathrm{Ph}), 128.26$ $(\mathrm{Ph}), 118.64(\mathrm{Ph}), 115.22(\mathrm{Ph}), 115.15(\mathrm{Ph}), 100.74$ (硻二 唑环), $55.97\left(\mathrm{OCH}_{3}\right), 24.80\left(\mathrm{CH}_{3}\right), 22.47\left(\mathrm{CH}_{3}\right), 18.51$ $\left(\mathrm{CH}_{3}\right), 18.32\left(\mathrm{CH}_{3}\right)$; IR (KBr) v: 3030, 2931, 2850, 1701, $1600,1526,1453,1257,820 \mathrm{~cm}^{-1}$; HRMS (positive-ESIMS) calcd for $\mathrm{C}_{16} \mathrm{H}_{17} \mathrm{~N}_{3} \mathrm{O}_{3} \mathrm{Se}(\mathrm{M}+1)^{+} 379.0435$, found 379.0430 .

3.3.11 (2-(4-氯苯基)-5-(2-(4-甲氧苯基)-4-甲基-1,3硒唑-5-基)-1,3,4-噁二唑-3(2H)-基)乙烯酮(SAZ-11)的 合成

取 $0.43 \mathrm{~g}(0.001 \mathrm{~mol}) \mathbf{S A - 8}$ 于三颈瓶中, 加入 $8 \mathrm{~mL}$ 乙酸酕, 搅拌至完全溶解, 回流, 用 TLC 监测反应, 反 应时间约为 $5 \mathrm{~h}$. 反应停止后, 冷却至室温, 将混合物倒 入 $50 \mathrm{~mL}$ 冰水混合物中, 搅拌, 有黄绿色固体析出, 抽 滤, 固体粗产品用丙酮重结晶得黄绿色固体 $0.291 \mathrm{~g}$, 收 率为 $61.25 \%$. m.p. $279.4 \sim 281.1{ }^{\circ} \mathrm{C} ;{ }^{1} \mathrm{H}$ NMR $(500 \mathrm{MHz}$, DMSO- $\left.d_{6}\right) \delta: 12.95(\mathrm{~s}, 1 \mathrm{H}, \mathrm{CH}), 7.90$ (d, $J=8.70 \mathrm{~Hz}, 2 \mathrm{H}$, $\mathrm{PhH}), 7.52$ (s, 2H, PhH), 7.19 (s, 2H, PhH), 7.05 (d, $J=$ $8.60 \mathrm{~Hz}, 2 \mathrm{H}, \mathrm{PhH}), 3.83$ (s, $\left.3 \mathrm{H}, \mathrm{OCH}_{3}\right), 2.63\left(\mathrm{~s}, 3 \mathrm{H}, \mathrm{CH}_{3}\right)$, $2.23\left(\mathrm{~s}, 3 \mathrm{H}, \mathrm{CH}_{3}\right) ;{ }^{13} \mathrm{C}$ NMR (125 MHz, DMSO- $\left.d_{6}\right) \delta$ : 174.52 (硒唑环), $167.20(\mathrm{C}=\mathrm{O}), 162.40$ (硒唑环), 157.37
(硒唑环), 152.60 (噁二唑环), $135.80(\mathrm{Ph}), 135.07(\mathrm{Ph})$, $129.40(\mathrm{Ph}), 129.25(\mathrm{Ph}), 129.04(\mathrm{Ph}), 128.19(\mathrm{Ph}), 117.95$ $(\mathrm{Ph}), 115.25(\mathrm{Ph}), 91.64$ (啞二㟇环), $56.01\left(\mathrm{OCH}_{3}\right), 21.62$ $\left(\mathrm{CH}_{3}\right), 18.20\left(\mathrm{CH}_{3}\right)$; IR (KBr) v: 3030, 2924, 1665, 1640, 1600, 1446, 1250, $835 \mathrm{~cm}^{-1}$; HRMS (positive-ESIMS) calcd for $\mathrm{C}_{21} \mathrm{H}_{18} \mathrm{ClN}_{3} \mathrm{O}_{3} \mathrm{Se}(\mathrm{M}+1)^{+}$475.0202, found 475.0198 .

3.3.12 2-(4-氯苯基)-5-(2-(4-甲氧苯基)-4-甲基-1,3硒唑-5-基)-1,3,4-悪二唑(SAZ-12)的合成

取 $0.31 \mathrm{~g}(0.001 \mathrm{~mol})$ 中间体 $\mathbf{S A}-\mathbf{4}$ 于三颈瓶中, 加入 $5 \mathrm{~mL}$ 三氯氧磷, 温度升至 $90{ }^{\circ} \mathrm{C}$, 搅拌至完全溶解后, 加入 $0.12 \mathrm{~g}(0.0011 \mathrm{~mol})$ 对氯苯甲酸, 搅拌至完全溶解, $30 \mathrm{~min}$ 后, 逐渐有黄绿色固体析出. 用 TLC 监测反应, 反应时间为 $7 \mathrm{~h}$. 反应停止后, 将混合物倒入 $60 \mathrm{~mL}$ 冰水 混合物中搅拌, 有黄绿色固体析出, 抽滤, 固体粗产品 用乙醇重结晶得浅黄色固体 $0.301 \mathrm{~g}$, 收率为 $70 \%{ }^{[53]}$. m.p. $252.3 \sim 253.6{ }^{\circ} \mathrm{C} ;{ }^{1} \mathrm{H}$ NMR (500 MHz, DMSO- $\left.d_{6}\right) \delta$ : $7.86(\mathrm{~d}, J=8.60 \mathrm{~Hz}, 4 \mathrm{H}, \mathrm{PhH}), 7.04$ (d, $J=8.75 \mathrm{~Hz}, 4 \mathrm{H}$, $\mathrm{PhH}), 3.83$ (s, 3H, $\left.\mathrm{OCH}_{3}\right), 2.56\left(\mathrm{~s}, 3 \mathrm{H}, \mathrm{CH}_{3}\right) ;{ }^{13} \mathrm{C} \mathrm{NMR}$ (125 MHz, DMSO- $d_{6}$ ) $\delta: 172.94$ (硒唑环), 163.31 (硒唑 环), 161.96 (硒唑环), 155.03 (噁二唑环), 150.18 (噁二唑 环), $128.87(\mathrm{Ph}), 128.63(\mathrm{Ph}), 115.13(\mathrm{Ph}), 55.93\left(\mathrm{OCH}_{3}\right)$, $18.32\left(\mathrm{CH}_{3}\right)$; IR (KBr) v: 3092, 2924, 1686, 1592, 1483, $1250,828,762 \mathrm{~cm}^{-1}$; HRMS (positive-ESIMS) calcd for $\mathrm{C}_{19} \mathrm{H}_{14} \mathrm{ClN}_{3} \mathrm{O}_{2} \mathrm{Se}(\mathrm{M}+1)^{+} 430.9940$, found 430.9950 .

3.3.13 5-(2-(4-甲氧苯基)-4-甲基-1,3-硒唑-5-基)1,3,4-噻二唑-2-胺(SAZ-13)的合成

在 $0{ }^{\circ} \mathrm{C}$ 下, 取 $0.37 \mathrm{~g}(0.001 \mathrm{~mol})$ 中间体 $\mathbf{S A - 7}$ 于三颈 瓶中, 向体系中缓慢滴加 $5 \mathrm{~mL}$ 浓硫酸, 搅拌, 溶液为绿 色粘稠状液体, 滴加完毕, 继续反应 $30 \mathrm{~min}$, 将反应体 系转移至室温, 继续反应 $2.5 \mathrm{~h}$. 反应停止后, 将混合物 倒入 $40 \mathrm{~mL}$ 冰水中, 搅拌, 并用浓氨水调节 $\mathrm{PH}$ 值约为 7, 有黄绿色固体析出, 抽滤, 固体粗产品用乙醇重结晶 得黄绿色固体 $0.263 \mathrm{~g}$, 收率为 $75 \%$. m.p. 277.6 $278.8{ }^{\circ} \mathrm{C} ;{ }^{1} \mathrm{H}$ NMR (500 MHz, DMSO- $\left.d_{6}\right) \delta: 7.90 \sim 7.86$ (m, 2H, PhH), 7.46 (s, 2H, NH $), 7.05(\mathrm{t}, J=8.80 \mathrm{~Hz}, 2 \mathrm{H}$, $\mathrm{PhH}), 3.83$ (s, 3H, $\mathrm{OCH}_{3}$ ), 2.65 (s, $\left.3 \mathrm{H}, \mathrm{CH}_{3}\right) ;{ }^{13} \mathrm{C} \mathrm{NMR}$ (125 MHz, DMSO- $d_{6}$ ) $\delta: 174.96$ (硒唑环), 162.48 (硒唑 环), 158.27 (噻二唑环), 157.08 (噻二唑环), $129.28(\mathrm{Ph})$, $127.97(\mathrm{Ph}), 116.34(\mathrm{Ph}), 115.17(\mathrm{Ph}), 55.99\left(\mathrm{OCH}_{3}\right)$, $18.42\left(\mathrm{CH}_{3}\right)$; IR (KBr) v: 3426, 3030, 2924, 1665, 1600, 1520, 1432, 1286, 842, $667 \mathrm{~cm}^{-1}$; HRMS (positive-ESIMS) calcd for $\mathrm{C}_{13} \mathrm{H}_{12} \mathrm{~N}_{4} \mathrm{OSSe}(\mathrm{M}+1)^{+} 351.9897$, found 351.9910 . 
3.3.14 5-(2-(4-甲氧苯基)-4-甲基-1,3-硒唑-5-基)- $N$ 苯基- 1,3,4-噻二唑-2-胺(SAZ-14) 的合成

在 $0{ }^{\circ} \mathrm{C}$ 下, 取 $0.478 \mathrm{~g}(0.001 \mathrm{~mol})$ 中间体 $\mathbf{S A - 5}$ 于三 颈瓶中, 向体系中逐滴加入 $4 \mathrm{~mL}$ 浓硫酸, 搅拌, 溶液为 绿色粘稠状液体, 滴加完毕后, 继续搅拌 $30 \mathrm{~min}$, 将反 应体系转移至室温, 继续摚拌 $2.5 \mathrm{~h}$. 反应停止后, 将混 合物倒入 $50 \mathrm{~mL}$ 冰水中, 搅拌, 并用浓氨水调节 PH 值 约为 7 , 继续搅拌, 有黄绿色固体析出. 将固体抽滤, 固 体粗产品用乙醇重结晶得黄绿色固体 $0.306 \mathrm{~g}$, 收率为 71.4\%. m.p. $282.5 \sim 284.2{ }^{\circ} \mathrm{C}$; ${ }^{1} \mathrm{H}$ NMR $(500 \mathrm{MHz}$, DMSO- $\left.d_{6}\right) \delta: 0.68$ (s, 1H, NH), 7.90 (d, $J=8.45 \mathrm{~Hz}, 2 \mathrm{H}$, $\mathrm{PhH}), 7.65$ (d, $J=7.80 \mathrm{~Hz}, 2 \mathrm{H}, \mathrm{PhH}), 7.39$ (t, $J=7.80 \mathrm{~Hz}$, 2H, PhH), 7.06 (q, $J=8.45 \mathrm{~Hz}, 3 \mathrm{H}, \mathrm{PhH}), 3.84$ (s, 3H, $\left.\mathrm{OCH}_{3}\right), 2.62\left(\mathrm{~s}, 3 \mathrm{H}, \mathrm{CH}_{3}\right) ;{ }^{13} \mathrm{C} \mathrm{NMR}(125 \mathrm{MHz}$, DMSO- $\left.d_{6}\right) \delta: 173.26$ (硒唑环), 169.95 (硒唑环), 162.23 (硒唑环), 155.21 (噻二唑环), 154.18 (噻二唑环), 138.95 $(\mathrm{Ph}), 129.57(\mathrm{Ph}), 129.16(\mathrm{Ph}), 128.28(\mathrm{Ph}), 122.44(\mathrm{Ph})$, $117.81(\mathrm{Ph}), 117.58(\mathrm{Ph}), 115.20(\mathrm{Ph}), 55.98\left(\mathrm{OCH}_{3}\right)$, $18.26\left(\mathrm{CH}_{3}\right)$; IR (KBr) v: 3194, 3047, 2917, 1640, 1606, 1500, 1450, 1270, 747, $682 \mathrm{~cm}^{-1}$; HRMS (positive-ESIMS) calcd for $\mathrm{C}_{19} \mathrm{H}_{16} \mathrm{~N}_{4} \mathrm{OSSe}(\mathrm{M}+1)^{+} 428.0210$, found 428.0220 .

3.3.15 2-(4-甲氧苯基)-4-甲基- $N^{\prime}$-(4-羰基-3-苯基噻 唑烷-2-亚基)-1,3-硒唑-5-酰肼(SAZ-15)的合成

取 $0.478 \mathrm{~g}$ (0.001 mol)中间体 SA-5 于三颈瓶中, 加 入 $10 \mathrm{~mL}$ 的绝对无水乙醇搅拌溶解, 温度升至 $78{ }^{\circ} \mathrm{C}$, 再加入 $0.167 \mathrm{~mL}(0.001 \mathrm{~mol})$ 溴乙酸乙酯, 搅拌充分后, 加入 $4.5 \mathrm{~g}(0.01 \mathrm{~mol})$ 无水乙酸钠, 搅拌, $5 \mathrm{~min}$ 后, 溶液 变浑浊, 有黄色固体析出, 继搅拌回流, 用 TLC 监测反 应, 反应时间约为 $6 \mathrm{~h}$. 反应停止后, 将混合物冷却至室 温, 倒入 $50 \mathrm{~mL}$ 冰水混合物中, 搅拌, 有固体析出, 将 固体抽滤, 得黄绿色固体 $0.389 \mathrm{~g}$, 收率为 $80 \%$. m.p. $235.3 \sim 236.1{ }^{\circ} \mathrm{C} ;{ }^{1} \mathrm{H}$ NMR (500 MHz, DMSO- $\left.d_{6}\right) \delta$ : 10.69 (s, 1H, NH), 7.93 (d, J=8.70 Hz, 2H, PhH), 7.61 (d, $J=7.75 \mathrm{~Hz}, 2 \mathrm{H}, \mathrm{PhH}), 7.39$ (t, $J=7.90 \mathrm{~Hz}, 2 \mathrm{H}, \mathrm{PhH}), 7.06$ (q, $J=7.90 \mathrm{~Hz}, 3 \mathrm{H}, \mathrm{PhH}), 4.27$ (s, 2H, $\left.\mathrm{CH}_{2}\right), 3.83$ (s, 3H, $\left.\mathrm{OCH}_{3}\right), 2.68\left(\mathrm{~s}, 3 \mathrm{H}, \mathrm{CH}_{3}\right) ;{ }^{13} \mathrm{C} \mathrm{NMR}(125 \mathrm{MHz}$, DMSO- $\left.d_{6}\right) \delta: 174.94$ (硒唑环), $166.48(\mathrm{C}=\mathrm{O}), 162.50$ (硒 唑环), 160.04 (硒唑环), 158.94 (噻唑烷环), 156.70 (噻唑 烷环), $137.58(\mathrm{Ph}), 130.28(\mathrm{Ph}), 129.89(\mathrm{Ph}), 129.38(\mathrm{Ph})$, $128.06(\mathrm{Ph}), 116.72(\mathrm{Ph}), 115.26(\mathrm{Ph}), 115.18(\mathrm{Ph}), 56.02$ $\left(\mathrm{OCH}_{3}\right), 44.20$ (噻唑烷环), $18.18\left(\mathrm{CH}_{3}\right)$; IR (KBr) v: 3233, 3055, 2975, 2844, 1738, 1665, 1600, 1475, 1250, 835, 690 $\mathrm{cm}^{-1}$; HRMS (positive-ESIMS) calcd for $\mathrm{C}_{21} \mathrm{H}_{18} \mathrm{~N}_{4} \mathrm{O}_{3} \mathrm{SSe}$ $(\mathrm{M}+1)^{+} 486.0265$, found 486.0259 .
3.3.16 $N^{\prime}$-(3,5-二苯基噻唑-2(3H)-亚基)-2-(4-甲氧苯 基)-4-甲基-1,3-硒唑-5-酰肼(SAZ-16)的合成

取 $0.478 \mathrm{~g}(0.001 \mathrm{~mol})$ 中间体 SA-5 于三口瓶中, 加 入 $12 \mathrm{~mL}$ 乙醇, 温度升至 $78{ }^{\circ} \mathrm{C}$, 搅拌, 不完全溶解, 体 系为浅绿色浊液, 加入 $0.148 \mathrm{~g}(0.0012 \mathrm{~mol})$ 2-溴苯乙酮, 搅拌, 体系由浅绿色浊液变为红棕色浊液, 搅拌 $30 \mathrm{~min}$ 后, 加入无水乙酸钠 $(0.002 \mathrm{~mol})$, 搅拌, 体系由红棕色 浊液变为黄色澄清溶液. 搅拌 $10 \mathrm{~min}$ 后, 有黄色固体析 出，继续搅拌回流，用 TLC 监测反应，反应时间约为 14 h. 反应停止后, 静置冷却至室温, 将混合物倒入冰水 中, 搅拌, 有黄绿色固体析出, 抽滤, 干燥, 将固体粗产 品用丙酮重结晶得黄绿色固体 $0.274 \mathrm{~g}$, 收率为 50.2\% ${ }^{[54]}$. m.p. 276.5 277.8 ${ }^{\circ} \mathrm{C}$; ${ }^{1} \mathrm{H}$ NMR $(500 \mathrm{MHz}$, DMSO-d $\left.d_{6}\right) \delta: 10.67$ (s, 1H, NH), 7.93 (d, $J=7.00 \mathrm{~Hz}, 2 \mathrm{H}$, $\mathrm{PhH}), 7.61$ (d, $J=7.80 \mathrm{~Hz}, 2 \mathrm{H}, \mathrm{PhH}), 7.39$ (t, $J=7.80 \mathrm{~Hz}$, $3 \mathrm{H}, \mathrm{PhH}), 7.26(\mathrm{~d}, J=4.60 \mathrm{~Hz}, 3 \mathrm{H}, \mathrm{PhH}), 7.07 \sim 7.01(\mathrm{~m}$, $4 \mathrm{H}, \mathrm{PhH}), 6.60(\mathrm{~s}, 1 \mathrm{H}, \mathrm{CH}=\mathrm{C}), 3.84(\mathrm{~d}, J=6.70 \mathrm{~Hz} 3 \mathrm{H}$, $\left.\mathrm{OCH}_{3}\right), 2.67\left(\mathrm{~s}, 3 \mathrm{H}, \mathrm{CH}_{3}\right) ;{ }^{13} \mathrm{C}$ NMR (125 MHz, DMSO$\left.d_{6}\right) \delta: 174.90$ (硒唑环), $166.48(\mathrm{C}=\mathrm{O}), 162.50$ (硒唑环), 160.04 (硒唑环), 158.94 (噻唑烷环), 156.76 (噻唑烷环), $137.52(\mathrm{Ph}), 130.28(\mathrm{Ph}), 129.86(\mathrm{Ph}), 129.36(\mathrm{Ph}), 128.06$ $(\mathrm{Ph}), 116.72(\mathrm{Ph}), 115.28(\mathrm{Ph}), 115.16(\mathrm{Ph}), 56.00\left(\mathrm{OCH}_{3}\right)$, $18.18\left(\mathrm{CH}_{3}\right)$; IR (KBr) v: 3348, 3048.3, 2931, 1700, 1665, 1600, 1490, 1257, $755 \mathrm{~cm}^{-1}$; HRMS (positive-ESIMS) calcd for $\mathrm{C}_{27} \mathrm{H}_{22} \mathrm{~N}_{4} \mathrm{O}_{2} \mathrm{SSe}(\mathrm{M}+1)^{+}$546.0629, found 546.0635 .

3.3.173-(2-(4-甲氧苯基)-4-甲基-1,3-硒唑-5-羰基)2-(苯基亚氨基)噻唑烷-4-酮(SAZ-17)的合成

在室温条件下, 取 $0.478 \mathrm{~g}(0.001 \mathrm{~mol})$ 中间体 SA-5 于三颈瓶中, 加入 $3 \mathrm{~mL}(0.01 \mathrm{~mol})$ 氯乙酰氯搅拌溶解, 体系为黄色溶液, 加入 $10 \mathrm{~mL} \mathrm{CHCl}_{3}$ 并将体系转入油浴 中，在 $60{ }^{\circ} \mathrm{C}$ 条件下继续反应，用 $\mathrm{TLC}$ 监测反应，反应 时间约为 $4 \mathrm{~h}$. 反应停止后，将混合物倒入 $50 \mathrm{~mL}$ 冰水混 合物中, 搅拌, 分液, 有机相干燥, 并蒸去溶剂, 固体用 $15 \mathrm{~mL}$ 无水乙醚洗涤 3 次, 得黄绿色固体 $0.230 \mathrm{~g}$, 收率 为 $50.2 \%$. m.p. $235.3 \sim 237.2{ }^{\circ} \mathrm{C}$; ${ }^{1} \mathrm{H}$ NMR $(500 \mathrm{MHz}$, DMSO- $\left.d_{6}\right) \delta: 7.94$ (d, $\left.J=8.80 \mathrm{~Hz}, 2 \mathrm{H}, \mathrm{PhH}\right), 7.56$ (q, $J=$ $7.70 \mathrm{~Hz}, 5 \mathrm{H}, \mathrm{PhH}), 7.06$ (t, J=8.80 Hz, 2H, PhH), 4.76 (s, $\left.2 \mathrm{H}, \mathrm{CH}_{2}\right), 3.83\left(\mathrm{t}, J=6.70 \mathrm{~Hz} 3 \mathrm{H}, \mathrm{OCH}_{3}\right), 2.57(\mathrm{~s}, 3 \mathrm{H}$, $\left.\mathrm{CH}_{3}\right) ;{ }^{13} \mathrm{C}$ NMR (125 MHz, DMSO- $\left.d_{6}\right) \delta: 174.97$ (硒唑 环), $166.48(\mathrm{C}=\mathrm{O}), 162.50$ (硒唑环), 160.03 (硒唑环), 158.99 (噻唑烷环), 156.76 (噻唑烷环), 137.56 (Ph), $130.28(\mathrm{Ph}), 129.89(\mathrm{Ph}), 129.38(\mathrm{Ph}), 128.05(\mathrm{Ph}), 116.71$ $(\mathrm{Ph}), 115.26(\mathrm{Ph}), 115.18(\mathrm{Ph}), 56.04\left(\mathrm{OCH}_{3}\right), 44.26$ (噻唑 烷环), $18.16\left(\mathrm{CH}_{3}\right)$; IR (KBr) v: 3034, 2931, 1723, 1640, 
$1600,1548,1446,1250,845 \mathrm{~cm}^{-1}$; HRMS (positive-ESIMS) calcd for $\mathrm{C}_{21} \mathrm{H}_{18} \mathrm{~N}_{2} \mathrm{O}_{3} \mathrm{SSe}(\mathrm{M}+1)^{+} 458.0203$, found 458.0200 .

3.3.18 5-(2-(4-甲氧苯基)-4-甲基-1,3-硒唑-5-基)- $1 H$ 四唑(SAZ-18)的合成

取 $0.28 \mathrm{~g}(0.001 \mathrm{~mol})$ 中间体 $\mathbf{S A - 1 1}$ 于三颈瓶中, 加 入 $6 \mathrm{~mL}$ DMF 搅拌至完全溶解, 加入 $0.107 \mathrm{~g}(0.002 \mathrm{~mol})$ 氯化铵摚拌完全溶解, 温度缓慢升至 $120{ }^{\circ} \mathrm{C}$, 加入 $0.013 \mathrm{~g}(0.001 \mathrm{~mol})$ 叠氮化钠, 搅拌, 用 TLC 监测反应, 反应时间约为 $3 \mathrm{~h}$, 反应完成后, 冷却至室温, 倒入冰水 混合物中, 搅拌, 有固体析出, 抽滤, 固体粗产品用无 水乙醇重结晶得浅黄色棒状晶体为 $0.210 \mathrm{~g}$, 收率为 $65 \%{ }^{[55]}$. m.p. $245.7 \sim 247.2{ }^{\circ} \mathrm{C} ;{ }^{1} \mathrm{H}$ NMR $(500 \mathrm{MHz}$, DMSO- $\left.d_{6}\right) \delta: 7.95(\mathrm{q}, J=9.00 \mathrm{~Hz}, 2 \mathrm{H}, \mathrm{PhH}), 7.07$ (d, $J=$ $7.70 \mathrm{~Hz}, 2 \mathrm{H}, \mathrm{PhH}), 3.84$ (s, 3H, $\left.\mathrm{OCH}_{3}\right), 2.71\left(\mathrm{~s}, 3 \mathrm{H}, \mathrm{CH}_{3}\right)$; ${ }^{13} \mathrm{C}$ NMR (125 MHz, DMSO- $d_{6}$ ) $\delta: 174.99$ (硒唑环), 162.46 (硒唑环), 157.00 (四唑环), $129.28(\mathrm{Ph}), 127.97$ $(\mathrm{Ph}), 116.34(\mathrm{Ph}), 115.17(\mathrm{Ph}), 55.99\left(\mathrm{OCH}_{3}\right), 18.42$ $\left(\mathrm{CH}_{3}\right)$; IR (KBr) v: 3326, 3035, 2924, 2203, 1600, 1520, $1461,1265,767 \mathrm{~cm}^{-1}$; HRMS (positive-ESIMS) calcd for $\mathrm{C}_{12} \mathrm{H}_{11} \mathrm{~N}_{5} \mathrm{OSe}(\mathrm{M}+1)^{+} 321.0129$, found 321.0124.

3.3.19 $N^{\prime}$-(2,5-二氧-2,5-二氢-1 $H$-吡咯-1-基)-2-(4-甲 氧苯基)-4-甲基-1,3-硒唑-5-甲酰胺(SAZ-19)的合成

取 $0.31 \mathrm{~g}(0.001 \mathrm{~mol})$ 中间体 $\mathbf{S A}-\mathbf{4}$ 于三颈瓶中, 加入 冰乙酸搅拌至完全溶解, 再加 $0.15 \mathrm{~g}(0.001 \mathrm{~mol})$ 顺丁烯 二酸酐搅拌完全溶解, 回流. 用 TLC 监测反应, 反应时 间约为 $10 \mathrm{~h}$. 反应停止后, 冷却至室温, 将混合物倒入 冰水中, 搅拌有固体析出, 抽滤, 固体粗产品用乙醇重 结晶得浅黄色棒状晶体 $0.254 \mathrm{~g}$, 收率为 $65 \%$. m.p. 165.2 167.0 ${ }^{\circ} \mathrm{C} ;{ }^{1} \mathrm{H}$ NMR (500 MHz, DMSO- $\left.d_{6}\right) \delta$ : $11.05(\mathrm{~s}, 1 \mathrm{H}, \mathrm{NH}), 8.03 \sim 8.01(\mathrm{~m}, 1 \mathrm{H}, \mathrm{CH}=\mathrm{CH}), 7.99 \sim$ $7.97(\mathrm{~m}, 1 \mathrm{H}, \mathrm{CH}=\mathrm{CH}), 7.95(\mathrm{~d}, J=8.75 \mathrm{~Hz}, 2 \mathrm{H}, \mathrm{PhH})$, 7.08 (d, $J=8.75 \mathrm{~Hz}, 2 \mathrm{H}, \mathrm{PhH}), 3.85$ (s, 3H, $\left.\mathrm{OCH}_{3}\right), 2.65$ $\left(\mathrm{s}, 3 \mathrm{H}, \mathrm{CH}_{3}\right) ;{ }^{13} \mathrm{C}$ NMR $\left(125 \mathrm{MHz}\right.$, DMSO- $\left.d_{6}\right) \delta: 175.13$ (硒唑环), 165.67 (硒唑环), 162.72 (硒唑环), $162.42(\mathrm{C}=$ O), $159.18(\mathrm{C}=\mathrm{O}), 135.95$ (吡咯环), 129.31 (吡咯环), $56.01\left(\mathrm{OCH}_{3}\right), 18.75\left(\mathrm{CH}_{3}\right)$; IR (KBr) v: 3222, 3084, 2967, 2830, 1730, 1650,1600, 1500, 1450, 1250, 820, $690 \mathrm{~cm}^{-1}$; HRMS (positive-ESIMS) calcd for $\mathrm{C}_{16} \mathrm{H}_{13} \mathrm{~N}_{3} \mathrm{O}_{4} \mathrm{Se}(\mathrm{M}+1)^{+}$ 391.0071, found 391.0069.

3.3.20 3-(2-(4-甲氧苯基)-4-甲基-1,3-硒唑-5-基)-5,6二苯基-1,2,4-三嗪(SAZ-20)的合成

向三颈瓶中加入 $0.0983 \mathrm{~g}\left(4.68 \times 10^{-4} \mathrm{~mol}\right)$ 联苯甲 酰和 $10 \mathrm{~mL}$ 乙醇, 搅拌, 温度升至 $78{ }^{\circ} \mathrm{C}$, 完全溶解后加 入 $0.145 \mathrm{~g}\left(4.68 \times 10^{-4} \mathrm{~mol}\right)$ 的中间体 $\mathbf{S A - 4}$, 再次完全溶
解后加入醋酸铵 $0.3605 \mathrm{~g}\left(4.68 \times 10^{-3} \mathrm{~mol}\right)$, 完全溶解后 加入草酸 $0.0118 \mathrm{~g}\left(4.68 \times 10^{-3} \mathrm{~mol}\right)$, 溶液由澄清变浑浊, 回流，用 $\mathrm{TLC}$ 监测反应，反应时间约为 $7 \mathrm{~h}$. 反应停止 后, 静置冷却至室温, 过滤, 得黄绿色的滤液, 蒸去溶

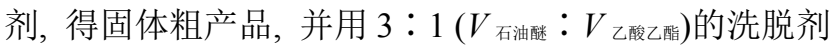
进行柱层析得黄绿色固体 $0.136 \mathrm{~g}$, 收率为 $60 \%$. m.p. $284.2 \sim 285.6{ }^{\circ} \mathrm{C} ;{ }^{1} \mathrm{H}$ NMR (500 MHz, DMSO- $d_{6}$ ) $\delta: 7.97$ (d, $J=8.00 \mathrm{~Hz}, 2 \mathrm{H}, \mathrm{PhH}), 7.89$ (q, $J=8.00 \mathrm{~Hz}, 2 \mathrm{H}, \mathrm{PhH}$ ), $7.67 \sim 7.63(\mathrm{~m}, 3 \mathrm{H}, \mathrm{PhH}), 7.60 \sim 7.54(\mathrm{~m}, 1 \mathrm{H}, \mathrm{PhH}), 7.49$ (d, $J=7.70 \mathrm{~Hz}, 2 \mathrm{H}, \mathrm{PhH}), 7.47 \sim 7.42(\mathrm{~m}, 2 \mathrm{H}, \mathrm{PhH}), 6.98$ (d, $J=7.70 \mathrm{~Hz}, 2 \mathrm{H}, \mathrm{PhH}), 3.88(\mathrm{~d}, J=4.30 \mathrm{~Hz}, 3 \mathrm{H}$, $\left.\mathrm{OCH}_{3}\right), 2.83\left(\mathrm{~s}, \quad 3 \mathrm{H}, \mathrm{CH}_{3}\right) ;{ }^{13} \mathrm{C} \quad \mathrm{NMR}(125 \mathrm{MHz}$, DMSO- $\left.d_{6}\right) \delta: 174.90$ (硒唑环), 162.50 (硒唑环), 160.04 (硒唑环), 158.98 (三嗪环), 156.78 (三嗪环), $137.52(\mathrm{Ph})$, $130.28(\mathrm{Ph}), 129.86(\mathrm{Ph}), 129.36(\mathrm{Ph}), 128.06(\mathrm{Ph}), 116.72$ $(\mathrm{Ph}), 115.28(\mathrm{Ph}), 115.16(\mathrm{Ph}), 56.00\left(\mathrm{OCH}_{3}\right), 18.18$ $\left(\mathrm{CH}_{3}\right)$; IR (KBr) v: 3157, 3047, 2924, 1621, 1600, 1510, 1450, 1250, 845, $690 \mathrm{~cm}^{-1}$; HRMS (positive-ESIMS) calcd for $\mathrm{C}_{26} \mathrm{H}_{20} \mathrm{~N}_{4} \mathrm{OSe}(\mathrm{M}+1)^{+}$484.0802, found 484.0808 .

3.3.21 N-(1,3-二氧代异吲哚啉-2-基)-2-(4-甲氧苯 基)-4-甲基-1,3-硒唑-5-甲酰胺(SAZ-21)的合成

取 $0.31 \mathrm{~g}(0.001 \mathrm{~mol})$ 中间体 $\mathbf{S A - 4}$ 于三颈瓶中, 加入 $10 \mathrm{~mL}$ 冰乙酸搅拌至完全溶解, 再加入 $0.2 \mathrm{~g}(0.001 \mathrm{~mol})$ 邻苯二甲酸酩摚拌完全溶解，回流. 用 TLC 监测反应, 反应时间约为 $10 \mathrm{~h}$. 反应停止后, 冷却至室温, 将混合 物倒入冰水中, 搅拌有固体析出, 抽滤, 固体用乙醇重 结晶, 得浅黄色棒状晶体 $0.322 \mathrm{~g}$, 收率为 $73 \%$. m.p. $227.8 \sim 229.1{ }^{\circ} \mathrm{C}$; ${ }^{1} \mathrm{H}$ NMR (500 MHz, DMSO- $d_{6}$ ) $\delta$ : 11.05 (s, $1 \mathrm{H}, \mathrm{NH}), 8.03 \sim 8.01(\mathrm{~m}, 2 \mathrm{H}, \mathrm{PhH}), 7.99 \sim 7.97$ (m, 2H, PhH), 7.95 (d, $J=8.70 \mathrm{~Hz}, 2 \mathrm{H}, \mathrm{PhH}), 7.08$ (d, $J=$ $8.75 \mathrm{~Hz}, 2 \mathrm{H}, \mathrm{PhH}), 3.85$ (s, $\left.3 \mathrm{H}, \mathrm{OCH}_{3}\right), 2.65$ (s, 3H, $\mathrm{CH}_{3}$ ); ${ }^{13} \mathrm{C}$ NMR (125 MHz, DMSO- $\left.d_{6}\right) \delta: 175,13$ (硒唑环), 165.67 (硒唑环), 162.72 (硒唑环), $162.42(\mathrm{C}=\mathrm{O}), 159.18$ (异吲哚啉环), $135.95(\mathrm{Ph}), 129.88(\mathrm{Ph}), 129.31(\mathrm{Ph})$, $128.26(\mathrm{Ph}), 125.57(\mathrm{Ph}), 124.40(\mathrm{Ph}), 115.26(\mathrm{Ph}), 56.01$ $\left(\mathrm{OCH}_{3}\right), 18.78\left(\mathrm{CH}_{3}\right)$; IR $(\mathrm{KBr}) v$ : 3207, 3034, 2975, 1738, 1643, 1600, 1520, 1432, 1250, $697 \mathrm{~cm}^{-1}$; HRMS (positiveESIMS) calcd for $\mathrm{C}_{20} \mathrm{H}_{15} \mathrm{~N}_{3} \mathrm{O}_{4} \mathrm{Se}(\mathrm{M}+1)^{+} 441.0228$, found 441.0230 .

3.3.22 N-(2-(4-甲氧苯基)-4-甲基-1,3-硒唑-5-基)-酰 胖一香豆素甲酮(SAZ-22)的合成

取 $0.41 \mathrm{~g}(0.001 \mathrm{~mol})$ 中间体 SA-12 于三颈瓶中, 加 入 $10 \mathrm{~mL}$ 乙醇摚拌并完全溶解, 温度升至 $78{ }^{\circ} \mathrm{C}$, 加入 水杨醛 $95 \mu \mathrm{L}(0.0015 \mathrm{~mol})$, 搅拌, 加入 2 滴冰乙酸, 摚 
拌约 $20 \mathrm{~min}$ 后, 有黄色固体析出, 继续回流, 用 TLC 监 测反应, 反应时间约 $8 \mathrm{~h}$. 反应停止后, 静置冷却至室 温, 将混合物倒入冰水中, 搅拌, 有黄色固体析出, 抽 滤, 固体粗产品用乙醇重结晶得黄色针状晶体 $0.382 \mathrm{~g}$, 收率为 79\%. m.p. 226.1 227.1 ${ }^{\circ} \mathrm{C} ;{ }^{1} \mathrm{H}$ NMR (500 MHz, DMSO- $\left.d_{6}\right) \delta: 11.88(\mathrm{~s}, 1 \mathrm{H}, \mathrm{NH}), 10.07$ (s, 1H, NH), 8.58 $(\mathrm{s}, 1 \mathrm{H}, \mathrm{CH}=\mathrm{C}), 7.96(\mathrm{q}, J=7.80 \mathrm{~Hz}, 3 \mathrm{H}, \mathrm{PhH}), 7.27$ (s, 1H, PhH), 7.07 (q, $J=8.80 \mathrm{~Hz}, 2 \mathrm{H}, \mathrm{PhH}), 6.95$ (t, $J=7.80$ $\mathrm{Hz}, 2 \mathrm{H}, \mathrm{PhH}), 3.84\left(\mathrm{~s}, 3 \mathrm{H}, \mathrm{OCH}_{3}\right), 2.76\left(\mathrm{~s}, 3 \mathrm{H}, \mathrm{CH}_{3}\right) ;{ }^{13} \mathrm{C}$ NMR (125 MHz, DMSO- $\left.d_{6}\right) \delta: 178.75$ (硒唑环), 163.94 (硒唑环), 162.13 (硒唑环), $157.12(\mathrm{C}=\mathrm{O}), 140.91$ (吡喃 环), $131.85(\mathrm{Ph}), 128.96(\mathrm{Ph}), 126.83(\mathrm{Ph}), 120.82(\mathrm{Ph})$, $120.20(\mathrm{Ph}), 116.71(\mathrm{Ph}), 115.18(\mathrm{Ph}), 55.93\left(\mathrm{OCH}_{3}\right)$, $20.48\left(\mathrm{CH}_{3}\right)$; IR (KBr) v: 3150, 3011, 2924, 2822, 1636, 1600, 1556, 1461, 1279, 820, $755 \mathrm{~cm}^{-1}$; HRMS (positive-ESIMS) calcd for $\mathrm{C}_{22} \mathrm{H}_{17} \mathrm{~N}_{3} \mathrm{O}_{5} \mathrm{Se}(\mathrm{M}+1)^{+} 483.0333$, found 483.0330 .

致谢 活性耖选试验由国家新药篮选中心协助测定, 在 此对他们的帮助表示诚挚的谢意.

辅助材料(Supporting Information) 所有目标化合物 的核磁共振氢谱和碳谱. 这些材料可以免费从本刊网站 (http://sioc-journal.cn/)上下载.

\section{References}

[1] Karegoudar, P.; Karthikeyan, M. S.; Prasad, D. J.; Mahalinga, M.; Holla, B. S.; Kumari, N. S. Eur. J. Med. Chem. 2008, 43(2), 261.

[2] Bharti, S. K.; Nath, G.; Tilak, R.; Singh, S. K. Eur. J. Med. Chem. 2010, 45(2), 651 .

[3] Mohammad, H.; Mayhoub, A. S.; Cushman, M.; Seleem, M. N. J. Antibiot. 2015, 68, 259.

[4] de Sa, N. P.; Lino, C. I.; Fonseca, N. C.; Borelli, B. M.; Ramos, J. P.; Souza-Fagundes, E. M.; Rosa, C. A.; Santos, D. A.; Oliveira, R. B.; Johann, S. Eur. J. Med. Chem. 2015, 102, 233.

[5] Chimenti, F.; Bizzarri, B.; Maccioni, E.; Secci, D.; Bolasco, A.; Fioravanti, R.; Chimenti, P.; Granese, A.; Carradori, S.; Rivanera, D.; Lilli, D.; Zicari, A.; Distinto, S. Bioorg. Med. Chem. Lett. 2007, 17(16), 4635.

[6] Youssef, A. M.; Malki, A.; Badr, M. H. Med. Chem. 2012, 8, 151.

[7] Łaczkowski, K. Z.; Misiura, K.; Switalska, M. Anti-Cancer Agents Med. Chem. 2014, 14, 1271.

[8] Taori, K.; Paul, V. J.; Luesch, H. J. Am. Chem. Soc. 2008, 130(6), 1806.

[9] Stadtman, T. C. Ann. N. Y. Acad. Sci. 2000, 899, 399.

[10] Metanis, N.; Keinan, E.; Dawson, P. E. J. Am. Chem. Soc. 2006, 128,16684 .

[11] Nishina, A.; Kimura, H.; Kozawa, K.; Sommen, G.; Nakamura, T.; Heimgartner, H.; Koketsu, M.; Furukawa, S. Toxicol. Appl. Pharmacol. 2011, 257(3), 388.

[12] Venardos, K. M.; Perkins, A.; Headrick, J. Curr. Med. Chem. 2007, 14(14), 1539.
[13] Sanmartin, C.; Plano, D.; Font, M. Curr. Med. Chem. 2011, 18(30), 4635 .

[14] Ninomiya, M.; Garud, D. R.; Koketsu, M. Coord. Chem. Rev. 2011, 255(23), 2968.

[15] Farag, A. M.; Mayhoub, A. S.; Barakat, S. E.; Bayomi, A. H. Bioorg. Med. Chem. 2008, 16(2), 881.

[16] Xia, Y.; Fan, C. D.; Zhao, B. X.; Zhao, J.; Shin, D. S.; Miao, J. Y. Eur. J. Med. Chem. 2008, 43(11), 2347.

[17] Xia, Y., Dong, Z. W.; Zhao, B. X.; Ge, X.; Meng, N.; Shin, D. S.; Miao, J. Y. Bioorg. Med. Chem. 2007, 15(22), 6893.

[18] Simlot, R.; Izydore, R. A.; Wong, O. T. J. Pharm. Sci. 1994, 83, 367.

[19] Kanagarajan, V.; Thanusu, J.; Ezhilarasi, M. R. Chem. Heterocycl. Compd. 2011, 47, 60.

[20] Mallakpour, S.; Rafiee, Z. Polym. Adv. Technol. 2008, 19(8), 1015.

[21] Mallakpour, S.; Rafiee, Z. Polym. Bull. 2008, 60(4), 507.

[22] Mallakpour, S.; Rafiee, Z. Polymer 2007, 48(19), 5530.

[23] Mallakpour, S.; Rafiee, Z. Synlett 2007 (8), 1255.

[24] Abu-Zaied, M. A. Z.; Nawwar, G. A. M.; Swellem, R. H.; El-Sayed, S. H. Pharmacol. Pharm. 2012, 3, 254.

[25] Sahu, V. K. R.; Singh, A. K.; Yadav, D. Int. J. ChemTech. Res. 2011, 3, 1362.

[26] Jain, S. K.; Yadav, A. K.; Nayak, P. Int. J. Pharm. Sci. Drug Res. 2011, 3, 230 .

[27] Ouyang, X.; Piatnitski, E. L.; Pattaropong, V.; Chen, X. L.; He, H. Y.; Kiselyov, A. S.; Velankar, A.; Kawakami, J.; Labelle, M.; Smith, L.; Lohman, J.; Lee, S. P.; Malikzay, A.; Fleming, J.; Gerlak, J.; Wang, Y.; Rosler, R. L.; Zhou, K.; Mitelman, S.; Camara, M.; Surguladze, D.; Doody, J. F.; Tuma, M. C. Bioorg. Med. Chem. Lett. 2006, 16(22), 1191.

[28] Lewis, J. R. Nat. Prod. Rep. 1999, 16, 389.

[29] Dorville, A.; McCort-Tranchepain, I.; Vichard, D.; Sather, W.; Maroun, R.; Ascher, P.; Roques, B. P. J. Med. Chem. 1992, 35(14), 2551.

[30] Jaen, J. C.; Wise, L. D.; Caprathe, B. W.; Tecle, H.; Bergmeier, S.; Humblet, C. C.; Heffner, T. G.; Meltzer, L. T.; Pugsley, T. A. J. Med. Chem. 1990, 33(1), 311.

[31] Clemence, F.; Martet, O. L.; Delevallee, F.; Benzoni, J.; Jonanen, A.; Jouquey, S.; Mouren, M.; Deraedt, R. J. Med. Chem. 1988, 31(7), 1453.

[32] Hargrave, K. D.; Hess, F. K.; Oliver, J. T. J. Med. Chem. 1983, 26(8), 1158.

[33] Tsuji, K.; Ishikawa, H. Bioorg. Med. Chem. Lett. 1994, 4(13), 1601.

[34] Bell, F. W.; Cantrell, A. S.; Hoberg, M.; Jaskunas, S. R.; Johansson, N. J.; Jordan, C. L.; Kinnick, M. D.; Lind, P.; MorinJr, J. M. J. Med. Chem. 1995, 38(25), 4929.

[35] My Hang, V. H.; Michael, D. C.; Thomas, J. M.; Modi, W. Proc. Natl. Acad. Sci. 2006, 103(27), 10322.

[36] My Hang, V. H. WO 2008054538, 2008 [Chem. Abstr. 2008, 148, 474992].

[37] Gao, H. X.; Shreeve, J. M. Chem. Rev. 2011, 111(11), 7377.

[38] Huang, M. Z.; Luo, F. X.; Mo, H. B.; Ren, Y. G.; Wang, X. G.; Ou, X. M.; Lei, M. X.; Liu, A. P.; Huang, L.; Xu, M. C. J. Agric. Food Chem. 2009, 57(20), 9585.

[39] Lawson, E. C.; Luci, D. K.; Ghosh, S.; Kinney, W. A.; Reynolds, C. H.; Qi, J.; Smith, C. E.; Wang, T. P.; Minor, L. K.; Haertlein, B. J.; Parry, T. J.; Damiano, B. P.; Maryanoff, B. E. J. Med. Chem. 2009, 52(23), 7432.

[40] Iwai, Y.; Takahashi, H.; Hatakeyama, D.; Motoshima, K.; Ishikawa, M.; Sugita, K.; Hashimoto, Y.; Harada, Y.; Itamura, S.; Odagiri, T.; Tashiro, M.; Sei, Y.; Yamaguchi, K.; Kuzuhara, T. Bioorg. Med. Chem. 2010, 18(14), 5379. 
[41] Kishida, K.; Aoyama, A.; Hashimoto, Y. Chem. Pharm. Bull. 2010, 58(11), 1525.

[42] Kolukisaoglu, Ü.; Wendler, C.; Goerdes, D. J. Enzyme Inhib. Med. Chem. 2010, 25(6), 876.

[43] Kaminski, K.; Obniska, J.; Wiklik, B.; Atamanyuk, D. Eur. J. Med. Chem. 2011, 46(8), 4634.

[44] Bansode, T. N.; Shelke, J. V.; Dongre, V. G. Eur. J. Med. Chem. 2009, 44(12), 5094.

[45] Huang, W. G.; Jiang, Y. Y.; Li, Q.; Li, J.; Li, J. Y.; Lu, W.; Cai, J. C. Tetrahedron 2005, 61(7), 1863.

[46] Sun, L. P.; Shen, Q.; Piao, H. H.; Ma, W. P.; Gao, L. X.; Zhang, W.; Nan, F. J.; Li, J.; Piao, H. R. Eur. J. Med. Chem. 2011, 46(9), 3630.

[47] Shi, L.; Yu, H. P.; Zhou, Y. Y.; Du, J. Q.; Shen, Q.; Li, J. Y.; Li, J. Acta Pharmacol. Sin. 2008, 29, 278.

[48] Cao, S.; Foster, C.; Lazo, J. S.; Kingston, D. G. I. Bioorg. Med. Chem. 2005, 13(17), 5094.
[49] Suzanne, F.; Stephen, J. E.; Anthony, F. H. J. Org. Chem. 2004, 69(14), 4663.

[50] Hua, G.; Li, Y.; Slawin, A. M. Z.; Woollines, J. D. Org. Lett. 2006 $8(23), 5251$

[51] Li, C.; Liu, J. C.; Li, Y. R.; Gou, C.; Zhang, M. L.; Liu, H. Y.; Li, X. Z.; Zheng, C. J.; Piao, H. R. Bioorg. Med. Chem. Lett. 2015, 25, 3052 .

[52] Puthiyapurayil, P.; Poojarya, B.; Chikkanna, C.; Buridipad, S. K. Eur. J. Med. Chem. 2012, 53, 203.

[53] Banerjee, A. G.; Das, N.; Shengule, S. A.; Srivastava, R. S.; Shrivastava, S. K. Eur. J. Med. Chem. 2015, 101, 81.

[54] Bondock, S.; Fadaly, W.; Metwally, M. A. Eur. J. Med. Chem. 2010, 45, 3692.

[55] Faria, J. V.; dos Santos, M. S.; Bernardino, A. M. R.; Becker, K. M.; Machado, G. M. C.; Rodrigues, R. F.; Canto-Cavalheiro, M. M.; Leon, L. L. Bioorg. Med. Chem. Lett. 2013, 23, 6310.

(Li, L.; Fan, Y.) 